San Jose State University

SJSU ScholarWorks

Master's Projects

Master's Theses and Graduate Research

Fall 2017

\title{
VIRTUALIZED BASEBAND UNITS CONSOLIDATION IN ADVANCED LTE NETWORKS USING MOBILITY- AND POWER-AWARE ALGORITHMS
}

Uladzimir Karneyenka

San Jose State University

Follow this and additional works at: https://scholarworks.sjsu.edu/etd_projects

Part of the Computer Sciences Commons

\section{Recommended Citation}

Karneyenka, Uladzimir, "VIRTUALIZED BASEBAND UNITS CONSOLIDATION IN ADVANCED LTE NETWORKS USING MOBILITY- AND POWER-AWARE ALGORITHMS" (2017). Master's Projects. 571.

DOI: https://doi.org/10.31979/etd.sg3g-dr33

https://scholarworks.sjsu.edu/etd_projects/571

This Master's Project is brought to you for free and open access by the Master's Theses and Graduate Research at SJSU ScholarWorks. It has been accepted for inclusion in Master's Projects by an authorized administrator of SJSU ScholarWorks. For more information, please contact scholarworks@sjsu.edu. 


\title{
ViRTUALIZEd BASEBANd UNits CONSOLIDATION IN AdVANCED LTE Networks USing Mobility- ANd Power-AWARe Algorithms
}

\author{
A Writing Project Report \\ Presented to \\ The Faculty of the Department of Computer Science \\ San Jose State University
}

\author{
In Partial Fulfillment \\ Of the Requirements for the Degree \\ Master of Science
}

By

Uladzimir Karneyenka

May 2017 
(C) 2017

Uladzimir Karneyenka

ALL RIGHTS RESERVED 


\begin{abstract}
Virtualization of baseband units in Advanced Long-Term Evolution networks and a rapid performance growth of general purpose processors naturally raise the interest in resource multiplexing. The concept of resource sharing and management between virtualized instances is not new and extensively used in data centers. We adopt some of the resource management techniques to organize virtualized baseband units on a pool of hosts and investigate the behavior of the system in order to identify features which are particularly relevant to mobile environment. Subsequently, we introduce our own resource management algorithm specifically targeted to address some of the peculiarities identified by experimental results.
\end{abstract}




\section{ACKNOWLEDGEMENTS}

I would like to express my gratitude to Dr. Melody Moh for being my project advisor and providing invaluable mentorship and help with organizing, focusing and seeing this project through. Also I want to thank the committee - Dr. Melody Moh, Dr. Teng Moh, and Dr. Robert Chun - for their time and much appreciated comments and suggestions. 


\section{Table of Contents}

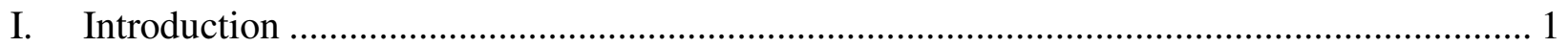

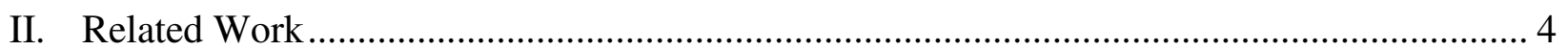

III. Clustering and Packing Algorithms ……………......................................................... 6

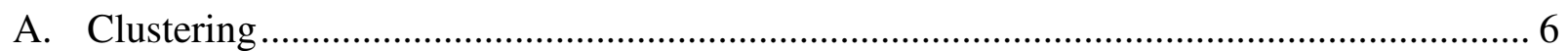

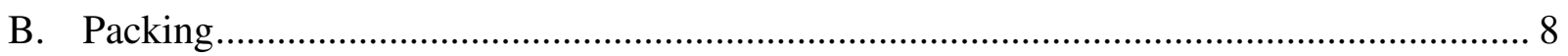

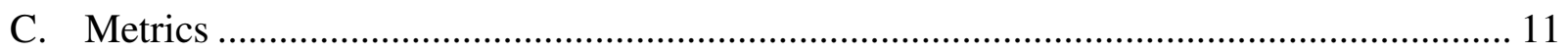

IV. Simulation and Experiments Setup ............................................................................. 13

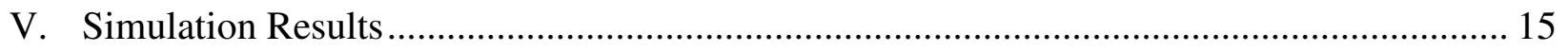

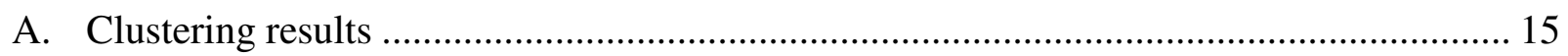

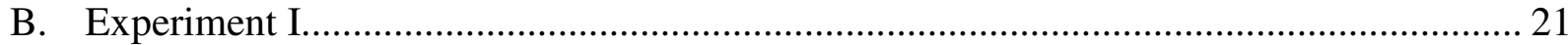

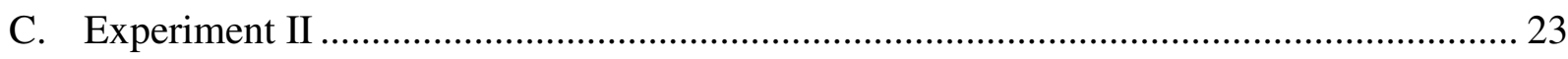

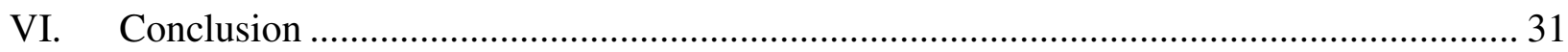

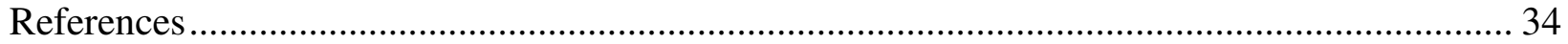

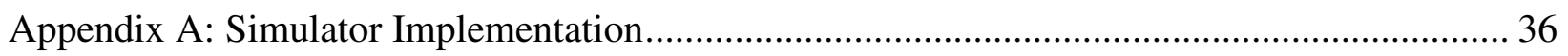




\section{List of Acronyms}

$\begin{array}{ll}\text { AP } & \text { Affinity Propagation (algorithm) } \\ \text { BBU } & \text { Base Band Unit } \\ \text { BFH } & \text { Best Fit Host (algorithm) } \\ \text { BS } & \text { Base Station } \\ \text { CoMP } & \text { Coordinated Multipoint } \\ \text { CPU } & \text { Central Processing Unit } \\ \text { FCC } & \text { Federal Communications Commission } \\ \text { FF } & \text { First Fit (algorithm) } \\ \text { GPP } & \text { General Purpose Platform } \\ \text { KVM } & \text { Kernel-based Virtual Machine } \\ \text { LTE } & \text { Long-Term Evolution } \\ \text { LXC } & \text { Linux Containers } \\ \text { QoS } & \text { Quality of Service } \\ \text { RM } & \text { Resource Manager } \\ \text { RRH } & \text { Remote Radio Head } \\ \text { UE } & \text { User Equipment } \\ \text { VBBU } & \text { Virtual Base Band Unit } \\ \text { VBS } & \text { Virtual Base Station } \\ \text { VM } & \text { Virtual Machine }\end{array}$




\section{INTRODUCTION}

The general trend in mobile devices usage shows persistent growth as the range of products that can make use of a cellular connectivity also increasing - phones, tablets, laptops, and vehicles. And now, the devices labeled as Internet of Things (IoT) are also getting introduced to the pool. This growing demand for connectivity requires service providers to react accordingly in order to accommodate not only coverage requirements, but also maintain a satisfactory Quality of Service (QoS); and for a service provider to stay competitive it has to invest into more sites. At the same time, various costs associated with deployment and operation of base stations (BS) are increasing each year; and the analysis of actual expenditures associated with a cellular site shows great spending not only in hardware, but also from utility and rental bills [16]. Another issue with a traditional cellular architecture worth noting is its rigidity which is due to hardware usually being vendor-locked. To some extent this constraint exacerbates any attempt to mix-andmatch the hardware and to upgrade or migrate to new standards and protocols [1].

When we talk about increasing number of mobile devices and the architecture that provides connectivity we also have to consider the "mobility" aspect. There is a notion of a "tidal effect" which can be inferred if we look at one's average weekday routine and then apply it to a population of any town or city [16]. An average person sleeps at night, gets up in the morning, commutes to the place of business, and then, towards the evening, goes back home. The researchers identified common trends for the residential, business, and entertainment areas drawing attention to the periods of prolonged time with the major energy waste when the cellular infrastructure is fully operational while having little demand. It should be stressed, a site providing connectivity must be operational $24 / 7$, and the same requirement stands when a service provider increases the number of sites in the area in order to accommodate the growing number of users. There are various research papers which address the energy problem for areas with densely populated cellular sites during "off-peak" hours. The major potential challenge applying those algorithms is to deploy them in a heavily vendor-locked infrastructure.

Increasing cost associated with deploying, operating, maintaining and scaling cellular networks led the researchers to investigate and propose a new architecture shifting away from a traditional distributed paradigm and embrace centralization [16]. The main concept is to move as much processing from remote sites as possible leaving just key functionality required for radio 
signal acquisition and broadcast. The proposed separation of functionality was applied on an evolved base station known as eNodeB from the Long-Term Evolution (LTE) communication standard, within which two main processing blocks were identified - Remote Radio Head (RRH) and Base Band Unit (BBU).
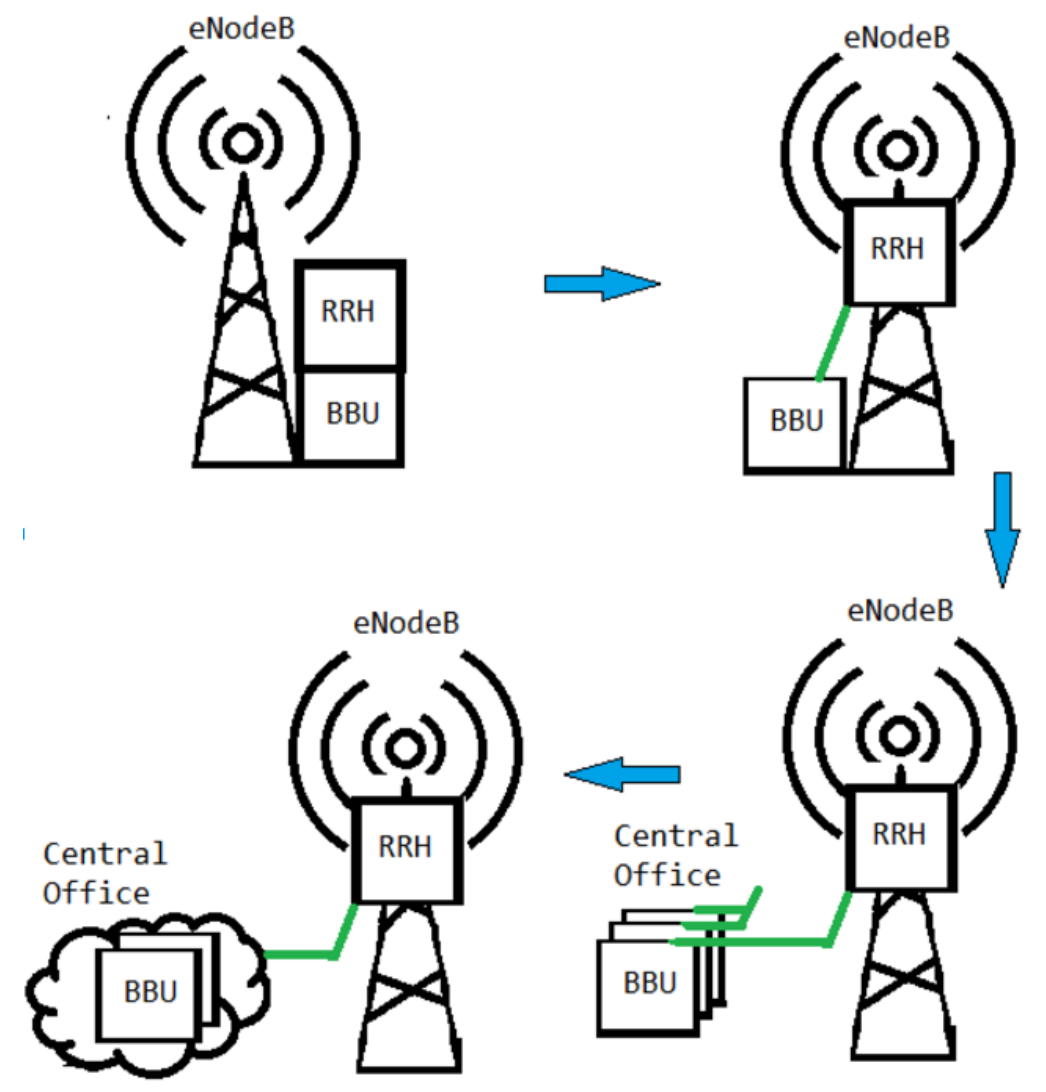

Fig. 1. Modularization and separation of eNodeB functionality.

$\mathrm{RRH}$ is responsible for functions required to transmit and receive radio signal, and BBU performs baseband processing along with other functionalities associated with L1, L2, and L3 layers from LTE protocol stack [1]. With the presence of a high-bandwidth low-latency link, such as fiber-optics communication, RRH and BBU can be distanced from each other by tens of kilometers without breaking stringent constraints specified by the LTE standard. Placing BBUs from multiple remote sites at a central location by itself reduces various costs and also enables migration from vendor-locked packages towards general purpose platforms (GPP) by implementing and running BBU in software. An ongoing research, implementation and evaluation of BBU realization in software shows that it is feasible to have BBU functionality hosted on GPP. Bhaumik et al. designed, implemented and tested their own framework based on 
OpenAirInterface project which allowed them to run multiple BBU instances on GPP [15]. The investigation by Nikaein on performance outlined some of the processing requirements for GPP to host BBU within various non- and virtualized host environments such as Virtual Machine $(\mathrm{KVM})$ and containers (Docker, LXC) [10]. Since running a BBU instance is very computationally demanding, to reduce performance hit on a host's CPU, a split BBU architecture can be applied, which originally was designed to alleviate inter-BBU communication load for cooperative scheme based scenarios [5]. Furthermore, this split allows mixing and matching various hosting platforms optimized for various performance tasks, e.g. memory intensive, CPU intensive, etc., and consequently taking advantage of heterogeneity of platforms which might occur due to the progress in computing architectures.

Virtualizing a BBU and running its instance on a GPP at a centralized location allows for hardware resource multiplexing and additionally gives researchers the opportunity to investigate whether power saving techniques applicable in cloud data centers can be adopted to manage $\mathrm{BBU}$ pool resources in a more energy efficient manner.

In this paper we are adapting some energy-efficient resource management algorithms from data centers and apply them on a BBU pool. Along with them, we designed and implemented our own packing algorithms specifically targeted to be applied on a centralized mobile wireless architecture. Also, similar to the work done by Zou et al. and Lee et al., a few clustering algorithms are considered for the experiments in order to apply the same concepts for energy conservation in an RRH pool [13][14]. In our case, we will be looking at both - BBU and RRH pools - since in a centralized architecture they are separated but still interdependent. We developed a high-level behavioral simulator which allowed us to simulate and investigate the behavior of BBUs, RRHs and mobile users in a centralized architecture when we apply various clustering and packing techniques. From these simulations we attempt to draw some guidelines for a resource manager to follow in order to run an energy-efficient, yet performance-conscious system.

This paper format is: Section II covers the background, followed by Section III, where we discuss clustering and packing algorithms used in the experiments. The simulator implementation is discussed in Section IV. Section V and Section VI cover the input details and 
simulation results respectively. And Section VII is a discussion and conclusion on acquired experimental results.

\section{RELATED WORK}

In a centralized cellular architecture there are two areas we are interested in for energy conservation - remote sites and BBU Pool. One of the ideas considered for manipulating energy consumption on remote sites is to monitor run-time load and change operational power state of individual towers provided sufficient density of sites serving the area. And for BBU Pool, various multiplexing and consolidation techniques on BBU instances can be applied assuming BBU implementation allows for virtualization.

The concept of manipulating power state of running towers is applicable for both centralized and traditional (decentralized) - cellular architectures, and some proposed schemes specifically target the latter in order to improve the energy efficiency within the existing infrastructure. Zou et al. propose a clustering algorithm to partition the area and monitor runtime load requirements in order to bring towers up or down, effectively keeping just enough operational towers to meet the demand [13]. The algorithm requires a full state view for all the sites involved and is specifically tailored for areas with a high volume of cellular sites which are underutilized during "off-peak" hours. A decentralized scheme is proposed by Lee et al. in their research where a message-passing algorithm is applied to solve the problem of identifying towers which can be switched to a dormant state [14]. Mobile devices are clustered around particular towers using a form of an affinity propagation algorithm, and then the towers that were found underutilized can be brought down. The results from experiments in a simulated environment allowed to draw an important conclusion that the aggregate power consumption for a multi-site area can be reduced when the activation of towers is demand-based. Along with the techniques which target underutilized sites, a concept to reduce the aggregate power consumption of densely placed towers during "peak" hours can be considered [5]. It requires a fine-grained tuning of a tower power output in order to provide just enough signal strength to cover smaller area of responsibility, this approach not only reduces inter-site interference, but potentially also improves energy efficiency.

To optimize a BBU pool's power consumption, consolidation techniques can be applied on virtualized BBUs to reduce the number of active hosts. We can borrow some insight on various 
techniques and algorithms used in cloud data centers where resource managers (RM) pack virtual machines on hosts using criteria such as computational, storage, bandwidth and other requirements. It should be noted that the timing requirements for data centers and BBU Pool are very different and while the former can allow to have an interruption of service for a few seconds, time required to migrate a VM, the latter has more real-time characteristics and whatever operations required to consolidate virtualized BBUs are subject to a strict timing.

When resource managers perform VM packing on hosts, they limit available resources which in turn can impair the performance of an individual VM if at some point it requires more resources due to some external stimulus. To accommodate fluctuations in resource demand, RM can take advantage of history- and pattern-based prediction methods and provision required resources more efficiently [7]. In conjunction with known bin-packing algorithms RM can consolidate VMs with fewer migrations and proactively meet the resource demand. Pompili et al. in their work also address packing within BBU Pool using history of data traffic and mobility where RM can make some assumptions about day-to-day usage patterns, perform location-based clustering, identify correlation between several areas and associated clusters, and pack them so cumulative resource usage would not exceed host's resource cap, e.g. packing together clusters which exhibit opposite utilization trends [5]. VMs can also be clustered together based on interVM traffic and subsequently consolidated on hosts using a bin-packing algorithm [8]. The main advantage of this approach is that it reduces inter-host bandwidth requirement and improves inter-VM bandwidth since VMs with an active cross-talk are more likely to be placed on the same host effectively reducing the hop count to zero. When BBU implementation is non-atomic, that is, there is a mechanism to initiate, preempt and control the input into a BBU instance, RM can map BBUs to hosts on-demand whenever the input or resources are available. Bhaumik et al. developed a framework that allows for resource pooling where BBU instances are mapped for processing using a cyclic scheduling algorithm whenever the input data frames become available [15]. This approach allows RM to enforce real-time characteristic of BBU instances by dropping any data frame that does not fit allotted time and prevent a schedule violation for consecutive time-slots in the queue. 


\section{Clunstering And PACKING Algorithms}

In this paper we are considering various power saving techniques and approaches adopted from data centers and derived from relevant research on centralized and decentralized cellular architectures. We use clustering algorithms to group closely related BBU instances, collect usage and mobility information for history- and pattern-based provisioning, and use several packing algorithms to consolidate virtual BBU instances on hosts in order to reduce active host count and improve energy efficiency within a BBU pool.

\section{A. Clustering}

Before we apply clustering on virtual BBUs (VBBU), we associate each RRH with a VBBU instance in a one-to-one mapping to form a virtual base station (VBS). Then we can cluster VBSs using different criteria, for example, based on RRH's location [5]. The main advantage of such clustering is that it can facilitate inter-VBS management such as handover handling or coordinated multipoint (CoMP) operations [1][4][9]. Once the clusters are formed, we can treat them as VMs and map them on hosts. Another clustering criterion we consider is the mobility information where we take into account traffic intensity between RRHs. Also, during clustering, the bandwidth cap on host can be considered to avoid grouping VBS instances which during "peak" hours might cause bandwidth starvation, although, for the purpose of this discussion, we assume a host is capable to handle the aggregate bandwidth from all its VBS instances.

Our default clustering algorithm is a variant of a greedy hierarchical clustering with a bounding condition being the inter-VBS distance restriction for all elements within the forming clusters. With some assumptions, such as the check at line 14, where the implementation can be optimized by employing some geometry, the complexity of this algorithm is $\mathrm{O}\left(\mathrm{n}^{2}\right)$, where $\mathrm{n}$ is the number of VBSs. We also make use of a grid structure which fetches neighboring VBSs much quicker instead of being forced to iterate through all VBS instances in order to check for proximity [13]. This grid structure is created during RRHs initialization and calculated only once. The grid breaks 2D area into cells to which we map enclosed RRHs. For a position of interest, we can acquire a corresponding cell and neighbors around it in a linear time and subsequently all of the associated RRHs. 


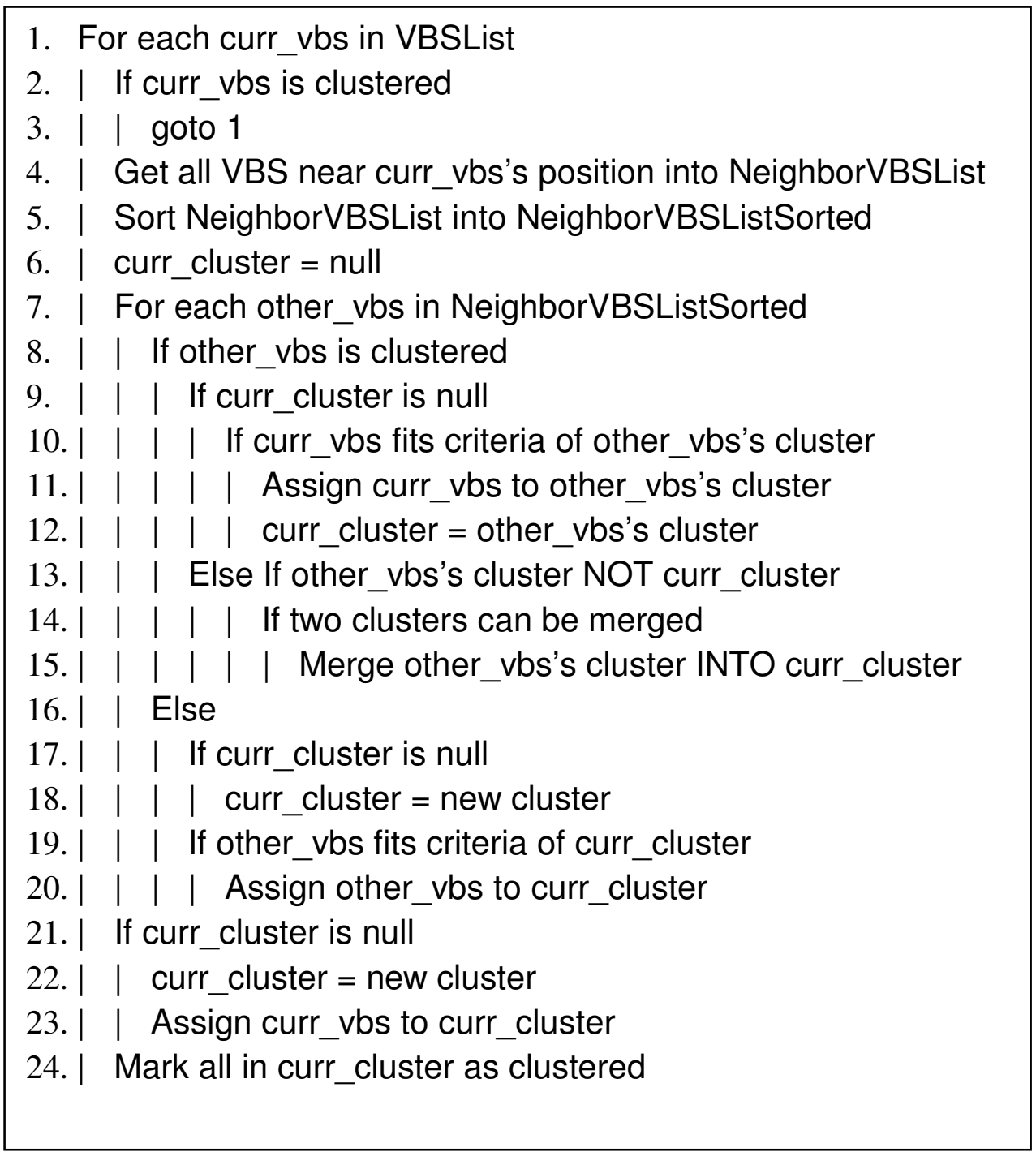

Fig. 2. Hierarchical clustering algorithm

Note: at line 4, we are getting all the neighbors around the position of curr_vbs, and that includes curr_vbs itself.

We also added another clustering algorithm - affinity propagation (AP) [6]. There are a few advantages to this algorithm over our default clustering scheme: a) it's a formal algorithm in contrast to our ad hoc default algorithm b) in a default AP implementation, the clusters are formed around actual points which we can safely designate as master nodes; c) to some extent we can modify clustering conditions in order to form clusters with specific properties, for example, we use the inter-RRH mobility information to gauge the intensity of users' movements 
from site to site and use it as a weight during AP cluster formulation which potentially can maximize handovers within cluster.

In order for our logical entities to have dynamic resource requirements we are grouping VBSs into cluster. We are making the same assumption regarding RRHs and assume that some RRHs can be put to sleep, while other RRHs in vicinity can be dynamically reconfigured to compensate the coverage in the affected areas [5][13][14],. So, when a particular tower is deactivated, in a centralized architecture, we can subsequently deactivate or otherwise reduce processing footprint inside BBU Pool, and if we have all VBSs clustered, the corresponding cluster's resource requirements decrease correspondingly. We are also using history-based prediction to proactively allocate or release resources used by VBS clusters [5][7]. To resize a VBS cluster we check each VBS instance for the immediate resource demand along with its historical data, and when some predetermined threshold is passed, the algorithm activates or deactivates VBS, effectively changing the amount of resources required.

\section{B. Packing}

Once clusters are established we can pack them based on runtime conditions to target particular performance goals such as throughput and energy conservation. We evaluate five packing algorithms:

1) Best Fit Host (BFH) algorithm which with the use of regression analysis packs VBS clusters on hosts and reduces the number of redundant migrations [7].

2) First-Fit $(F F)$ packing algorithm which requires less steps than BFH [8]. And we also make use of regression analysis which improves packing and prevents overutilization on hosts.

3) Mobility pattern (MP) based algorithm adopted from a packing concept discussed by Pompili et al. where the pattern is derived from usage history to identify pairs of clusters exhibiting reverse trends [5]. For example, a cluster for a residential area can be paired up with a cluster for a business area and if their usage pattern are reverse of each other they can be potentially packed on the same host effectively sharing resources and reducing further unnecessary migration.

4) Location-aware (LA) packing algorithm is the one we introduce in this paper. By default we form clusters using location-based principle which has a positive impact on any inter-VBS 
intensive task such as handovers. For the same reason, packing clusters together on the same host can improve the performance of VBSs' operations.

5) This is a modification of the location-aware packing, where we add into consideration the mobility factor between sites (LAM). We monitor and keep track of User Equipment (UE) movement and use history to measure the expected traffic intensity between cellular sites which translates into inter-cluster UE mobility, and we use it as a criterion (weights) to perform location-aware packing.

6) This is a modification of the location-aware packing algorithms above where we also consider the history of inter-RRH handovers (LAMH). 

1. Sort clusters by resources needed into ClustersSorted
2. Reverse sort hosts by utilization into HostSortedReversed
3. For curr_cluster in ClustersSorted
4. I If curr_cluster's host overultilized
5. | | Disassociate curr_cluster with its host
6. I If use mobility info
7. I | Reverse sort neighbors' hosts by mobility weights
8. I Else
9. I I Reverse sort neighbors' hosts by utilization
10. I For each neighbors' host
11. I I If current host is a fit
12. | | | Disassociate curr_cluster with its host
13. I | | Associate curr_cluster with current host
14. For curr_cluster in DisassociatedClusters
15. I If use mobility info
16. I I Reverse sort neighbors' hosts by mobility weights
17. I Else
18. I I Reverse sort neighbors hosts by utilization
19. I For each neighbors host
20. I I If current host is a fit
21. | | | Associate curr_cluster with current host
/* Handle host overutilization */
22. If DisassociatedClusters not empty
23. I Do bin-packing on DisassociatedClusters
/* Attempt to free up underutilized hosts /*
24. Sort hosts by utilization with new mapping into NewHostsSorted
25. For curr_host in NewHostsSorted
26. I Sort clusters on curr_host by utilization
27. I For each cluster on host
28. I I Do best fit packing

Fig. 3. Location-aware packing algorithm (with mobility)

Sorting on lines 7, 9, 16, and 18 can be done only once using available history. On lines 1 through 13, we are attempting to fit clusters on any host that has enough resources and an instance of any neighboring cluster. The condition statements on lines 6 and 15 enable the algorithm to pick the most promising neighbor based on mobility information. If there are clusters that were not associated with any host due to neighbors' hosts being packed or because 
originally it belonged to an overutilized host, the algorithm tries to pack them again with the same location-based and/or mobility criteria. After that, if there are still clusters left without a host, we apply a bin-packing algorithm which also activates new hosts on demand (lines 22-23). This effectively allows to address detected overutilization. Lines $24-28$ are there to pick any slack and attempt to free up any underutilized hosts by re-associating their clusters to other more heavily packed hosts that fit. Complexity breakdown of this algorithm with $\mathrm{M}$ representing the cluster count, and $\mathrm{N}-$ the host count, is:

- lines 3-13: $\mathrm{O}(\mathrm{MN})$

- lines 14-21: $\mathrm{O}(\mathrm{MN})$

- lines 22-23: $\mathrm{O}\left(\mathrm{M}^{2}\right)$

- lines 24-28: $\mathrm{O}(\mathrm{MN})$

The lines 22-23 of the algorithm are operating on a very small number of clusters, and the associated complexity has a very little effect on overall performance, so, for our proposed packing algorithm, we can assume the complexity to be of $\mathrm{O}(\mathrm{MN})$.

\section{Metrics}

Power

In centralized cellular architecture we have eNodeB broken into two components - RRH and BBU. An RRH has a smaller form factor than an eNodeB, requires less computational capacity, and, in some situations, can take advantage of a passive cooling altogether generating much smaller operational power footprint. A BBU, when moved to a centralized location such as a BBU pool, is most likely integrated into a centralized power and cooling infrastructure and we can only roughly estimate the impact it has on a BBU pool's power consumption. The power budget in a data center involves interdependent power components and runtime power takes a non-linear form [12]. In this paper we are only looking at computational components of a data center, and since we are assuming that our BBU pool takes form of a data center with VBBUs being hosted on GPPs, we borrow a power model elaborated by data centers researchers [7][17]:

$$
\begin{gathered}
E_{\text {total }}=E_{\text {comp }}+E_{\text {migr }}+E_{\text {swit }} \\
E_{\text {comp }}=\sum_{i=1}^{N} \int P_{c}\left(u_{i}(t)\right) d t
\end{gathered}
$$




$$
E_{m i g}=4 * \sum_{j=1}^{M} P_{m} \frac{C_{j}}{B W_{j}}
$$

, where the total energy (1) is the sum of the computational energy (2), the energy required to migrate VBBU instances between hosts (3), and the energy required to bring up hosts from a dormant state. For (2), $\mathrm{N}$ stands for the number of servers in consideration and $P_{c}\left(u_{i}(t)\right)$ is the power consumption by $i^{\text {th }}$ server with a utilization $\mathrm{u}_{i}(\mathrm{t})$. In (3), $\mathrm{P}_{m}$ stands for unit migration power consumption, $\mathrm{C}_{j}-$ migrated/transferred data size, and $\mathrm{BW}_{j}$ is the bandwidth used while migrating the $\mathrm{j}^{\text {th }}$ VM. Also, to simplify the power model, we are going to look at CPU utilization only and exclude any memory or storage power consumption from consideration. $\mathrm{E}_{\text {comp }}$ is a sum of all individual hosts' power consumption which we compute as functions of their corresponding CPU utilization. $E_{\text {migr }}$ is the power consumed to perform all the migrations which involves accounting for the size of data transmitted and the bandwidth. $\mathrm{E}_{\text {swit }}$ can be considered to be a sum of constants, especially in the case when the computing systems used in the pool are homogeneous.

\section{Migrations}

To analyze packing algorithms' performances we also look into the overall number of migrations. To move a VBBU instance time and resources are consumed, and, depending on the size of VBBUs and frequency they have to be migrated with, more load is added to the intraBBU pool network which can potentially introduce congestion and affect the quality of service.

\section{Handovers}

When mobile devices move between different areas of coverage within the cellular network, they have to undergo the process of transfer of their state from the tower they leave to the tower found in range. This process involves passing the corresponding UE context between towers, and in a centralized cellular architecture it can be facilitated if VBSs performing handovers are on the same host. On Figure 4, UE1 physically moves from the RRH1's coverage area into the RRH2's which causes the UE1's context to be transferred between corresponding VBBUs, and since they are sharing the same host it is less costly time- and resource-wise compared to the handover shown for UE2. 


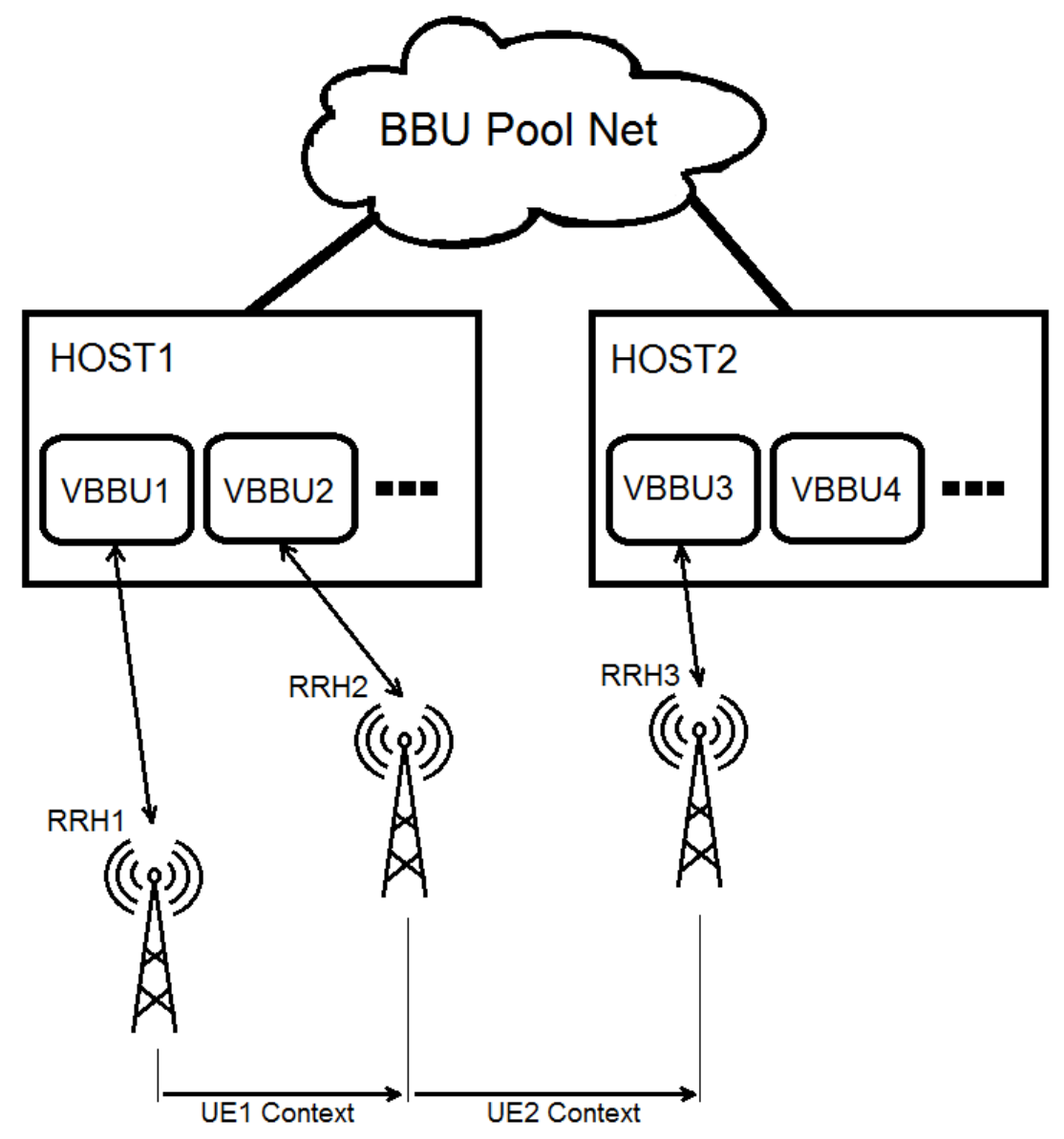

Fig. 4. Intra-BBU pool UE context transfer in a handover process.

\section{SimUlation AND EXPERIMENTS SETUP}

To run the simulation several input files have to be provided some of which are mandatory. A configuration file allows to specify several runtime constants describing host resources, algorithms to use, logging flags, etc. We also have to provide the placement of towers in the area and UEs' event file. In our simulator we have a functionality to generate a randomized set of UEs along with their bandwidth requirements and movement trajectories. This allows us to generate various UE sets following similar day-to-day pattern when we need to generate historical data for the prediction component. And to compare the algorithms in use, we generated and used the same set of UE events across multiple simulations.

Before the simulation, we place RRHs matching actual major tower positions for Bay Area available on the FCC website and then we arrange them into groups using three clustering algorithms which allow us to investigate how clustering affects resource management. 
TABLE I. Clustering algorithms used.

\begin{tabular}{|l|l|l|}
\hline Algorithm Name & Code & Complexity \\
\hline Hierarchical Clustering & HIER & $\mathrm{O}\left(\mathrm{r}^{2}\right)$ \\
\hline Affinity Propagation [6] & AP & $\mathrm{O}\left(\mathrm{r}^{2}\right)$ \\
\hline Affinity Propagation with Traffic & APT & $\mathrm{O}\left(\mathrm{r}^{2}\right)$ \\
\hline
\end{tabular}

TABLE II. Packing algorithms used.

\begin{tabular}{|l|l|l|}
\hline Algorithm Name & Code & Complexity \\
\hline Best Fit Host [7] & BFH & O(MN) \\
\hline First Fit [8] & FF & O(M log M) \\
\hline Mobility Pattern [5] & MP & O(M log M) \\
\hline Location aware & LA & O(MN) \\
\hline Location aware with Mobility & LAM & O(MN) \\
\hline Location aware with mobility and handovers & LAMH & O(MN $)$ \\
\hline
\end{tabular}

TABLE I and II list the clustering and packing algorithms used in the experiments with associated codes used in the graphs and the time complexity. For TABLE I, $r$ stands for the number of towers we are clustering, and for TABLE II M and N represent the number of clusters and hosts respectively. 


\section{Simulation Results}

\section{A. Clustering results}

Figure 5 shows the result of hierarchical clustering where the main criteria is the inter-RRH distance within clusters. The main purpose and advantage of this algorithm is the fact that all of the RRHs are equipotent and each can take up on a role of a master node, this, in turn, produces tighter clusters.

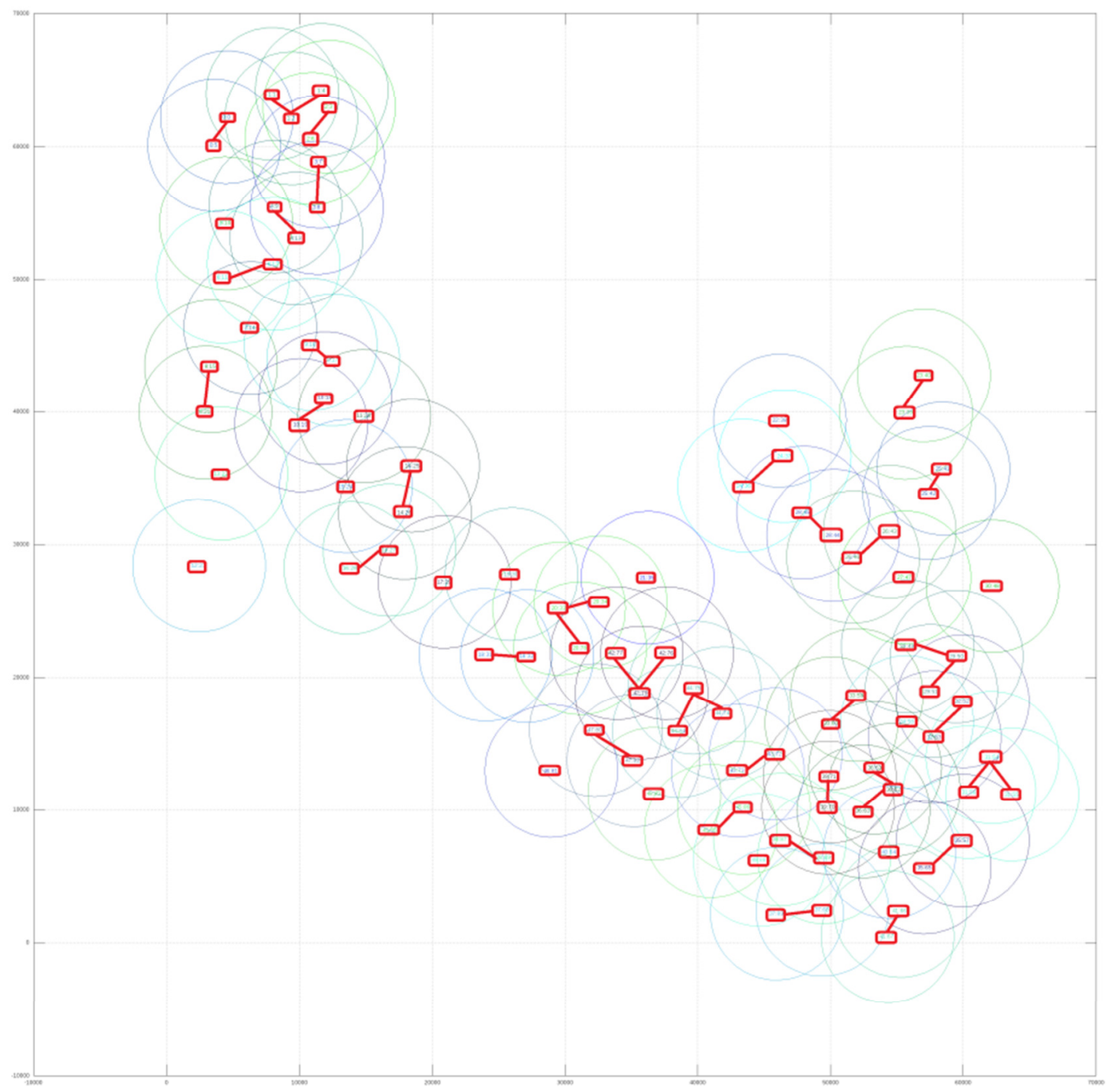

Fig. 5. Hierarchical clustering applied on mapped out RRHs. 
For the affinity propagation (AP) algorithm shown on Figure 6 we have a little bit lax distance constraint. In the default algorithm setup the clusters are formed around one of the points exemplars, - and that resulted in a spatially bigger clusters, and consequently, fewer clusters overall.

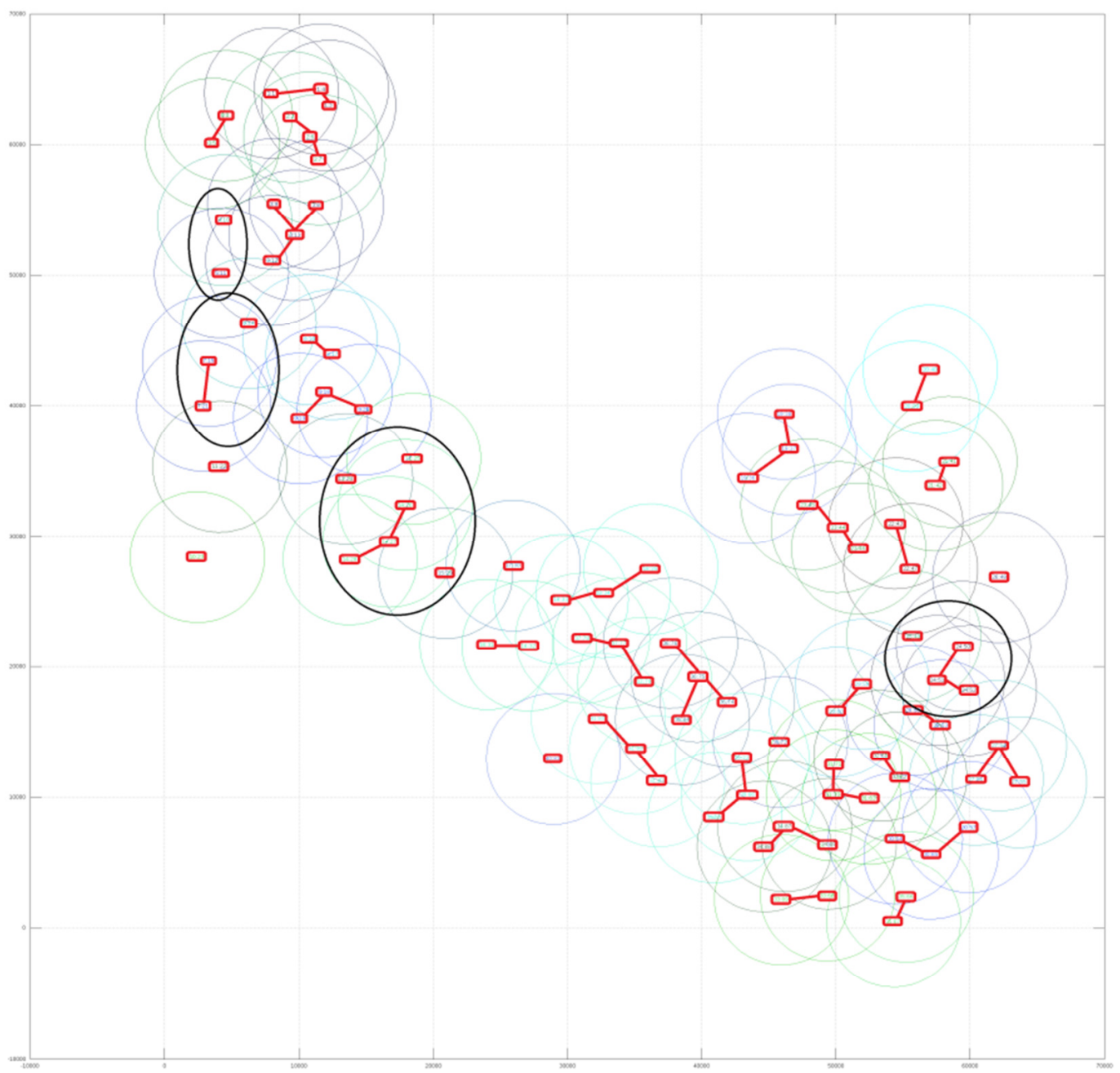

Fig. 6. Affinity propagation applied on mapped out RRHs. 
Additionally, we customized the AP algorithm by introducing weights into cluster consideration which allowed us flexibly apply slack on point-to-point distance constraint and bias edges with higher UE mobility. On Figure 7 we can see how those features affect some of the clusters (marked by ovals on both figures for AP), where new edges were added because of the slack and/or weights.

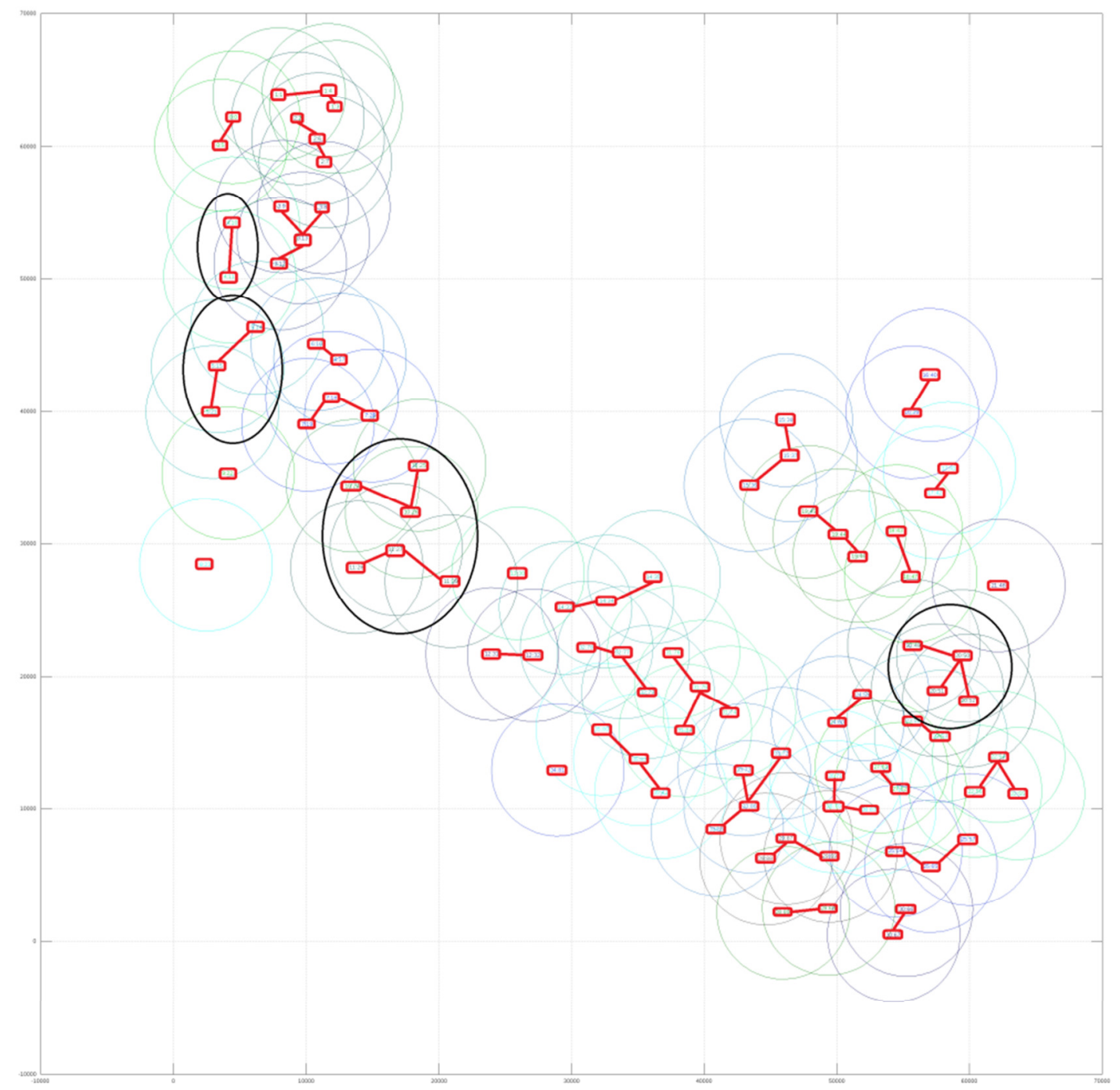

Fig. 7. Affinity propagation with traffic awareness applied on mapped out RRHs.

Due to different grouping goals used in the hierarchical clustering and affinity propagation algorithms, the resulting RRH distribution over clusters shows a significant imbalance biasing towards small clusters in the former. From TABLE III we can see how drastically the distribution of RRHs varies when applying different algorithms. It should be noted that it is the 
distance restriction in a hierarchical clustering and affinity propagation that contributes to this difference. We also see how the introduction of weights causes cluster count reduction in affinity propagation and reduces bias towards smaller clusters (1-RRH) even more.

TABLE III. RRH count breakdown across clusters.

\begin{tabular}{|l|l|l|l|}
\hline & $\begin{array}{l}\text { Hierarchical } \\
\text { clustering }\end{array}$ & $\begin{array}{l}\text { Affinity } \\
\text { propagation }\end{array}$ & $\begin{array}{l}\text { Affinity propagation } \\
\text { with traffic awareness }\end{array}$ \\
\hline Clusters & 50 & 42 & 36 \\
\hline 1-RRH Clusters & 17 & 13 & 5 \\
\hline 2-RRH Clusters & 26 & 12 & 12 \\
\hline 3-RRH Clusters & 7 & 15 & 15 \\
\hline 4-RRH Clusters & 0 & 2 & 4 \\
\hline
\end{tabular}

All the simulations share the same set of UE events which provides a stimulus to RRH clusters. And since we are experimenting with three clustering algorithms we have three sets of clusters' response. Every time a cluster resizes due to events from UEs, its resource utilization in the BBU-pool also gets affected subsequently inducing the resource manager to re-evaluate the resource requirements on corresponding host. The packing algorithms then re-arrange clusters on hosts to eliminate underprovisioning and to free the resources if re-packing allows for it.

Figures 8-10 show clusters fluctuations due to a moderate stimulus from UE input. There are steep rise and drop near the beginning and the end of the simulation, but in general, local runtime maximums coming from UE events show mild variation causing flatter waveform. In TABLE III the hierarchical clustering algorithm shows bias towards smaller clusters compared to AP-type algorithms, and the corresponding waveform on Figure 8 shows a little bit more aggressive response to the input.

These figures list "utilization" and "effective utilization" values which allow us to estimate the potential energy savings compared to a static use of RRH in the latter, and a static 
provisioning in the former, when the resource manager activates just enough RRHs to provide coverage for the predicted peak. The utilization percentages are based on area under the curve calculation. For example, the whole system has 90 RRHs installed, out of which approximately $84 \%$ are used in general throughout the simulation when hierarchical clustering is applied (Figure.8). The simulation also showed at the peak only $79 \mathrm{RRHs}$ are utilized, which is "effective" RRH count which the system needs to operate throughout the day, and "effective utilization" basically represents how much of those 79 RRHs were utilized during the simulation. So, "utilization" and "effective utilization" percentages shown on these figures are to represent the ratio of the area under the runtime curve to the areas under the maximum and runtime peak correspondingly.

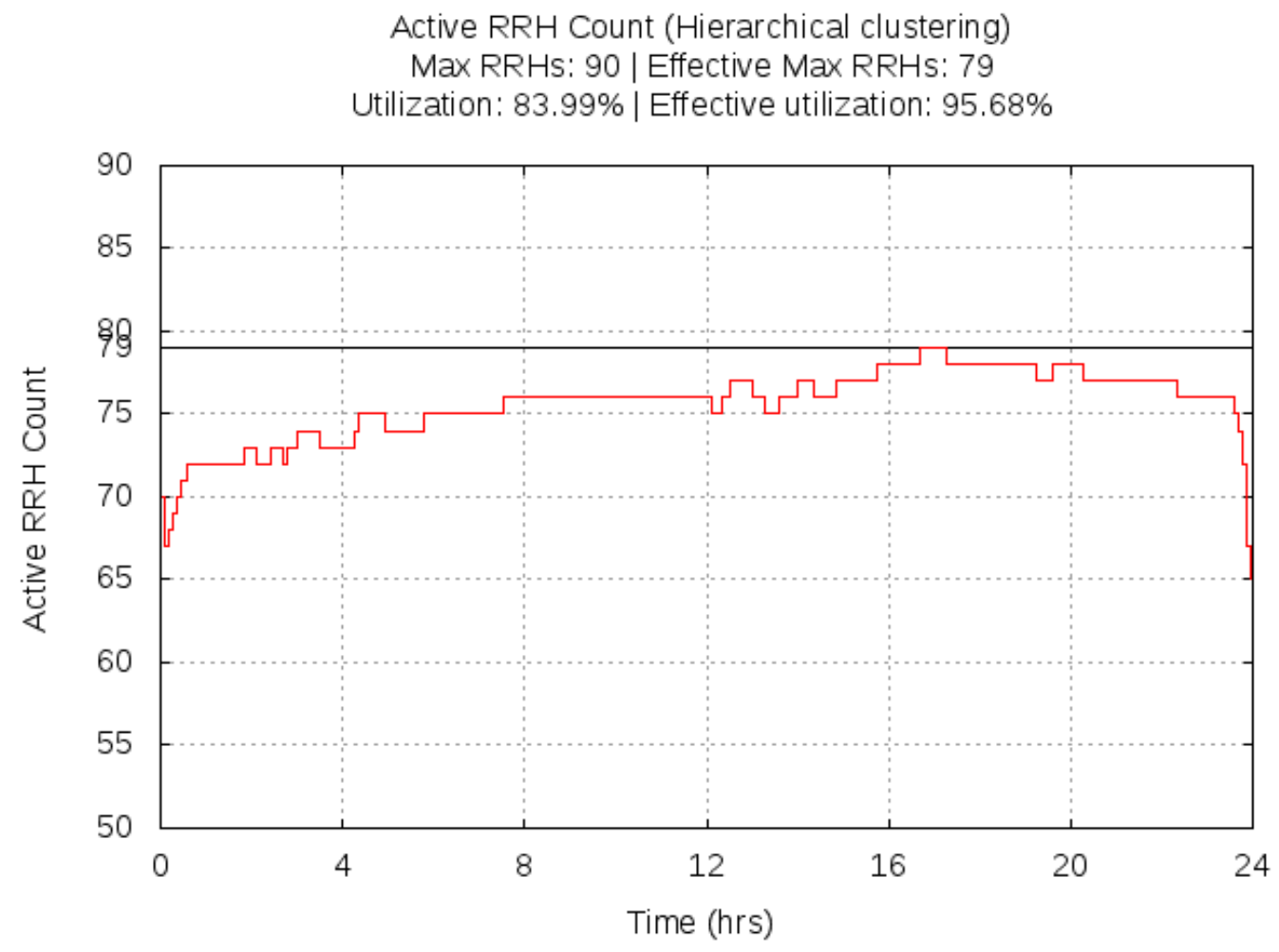

Fig. 8. Clusters fluctuation due to the hierarchical clustering. 
Active RRH Count (Affinity Propagation)

Max RRHs: 90 | Effective Max RRHs: 75

Utilization: $79.74 \%$ | Effective utilization: $95.69 \%$

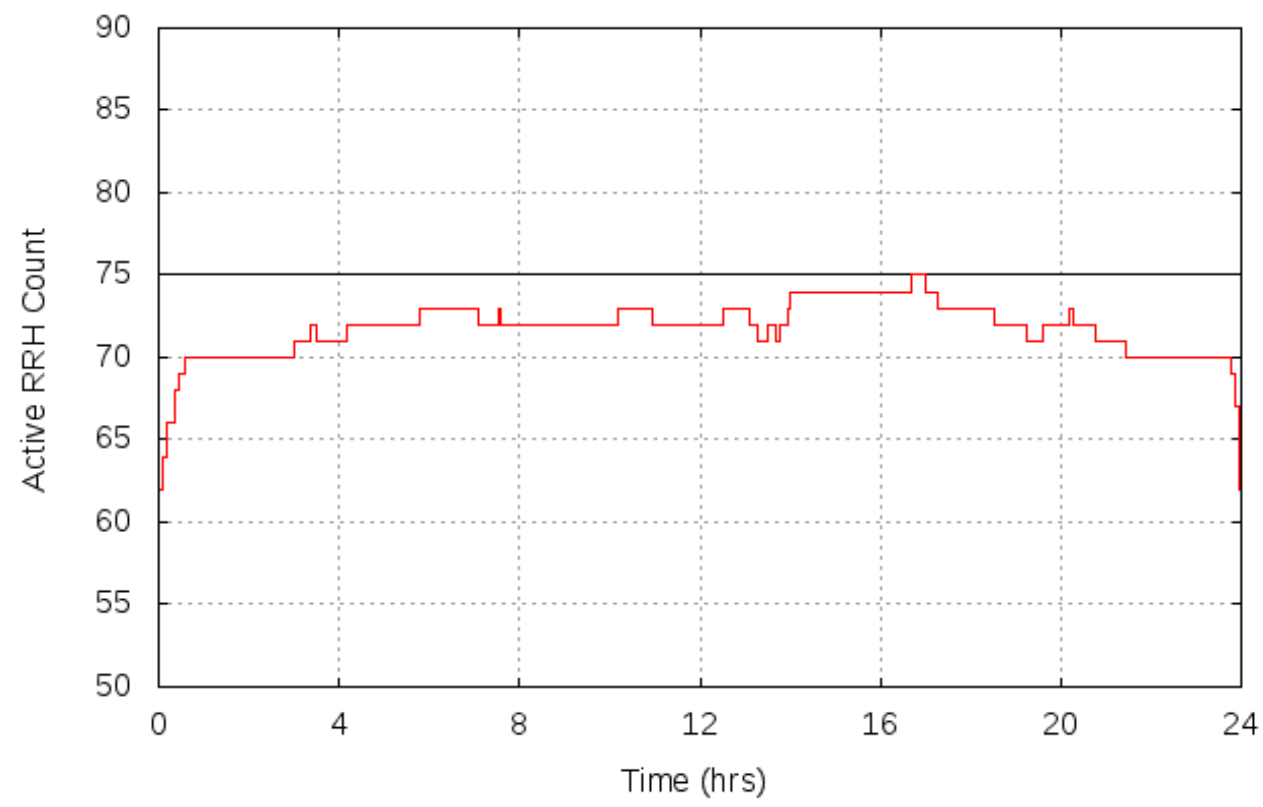

Fig. 9. Clusters fluctuation due to the affinity propagation clustering.
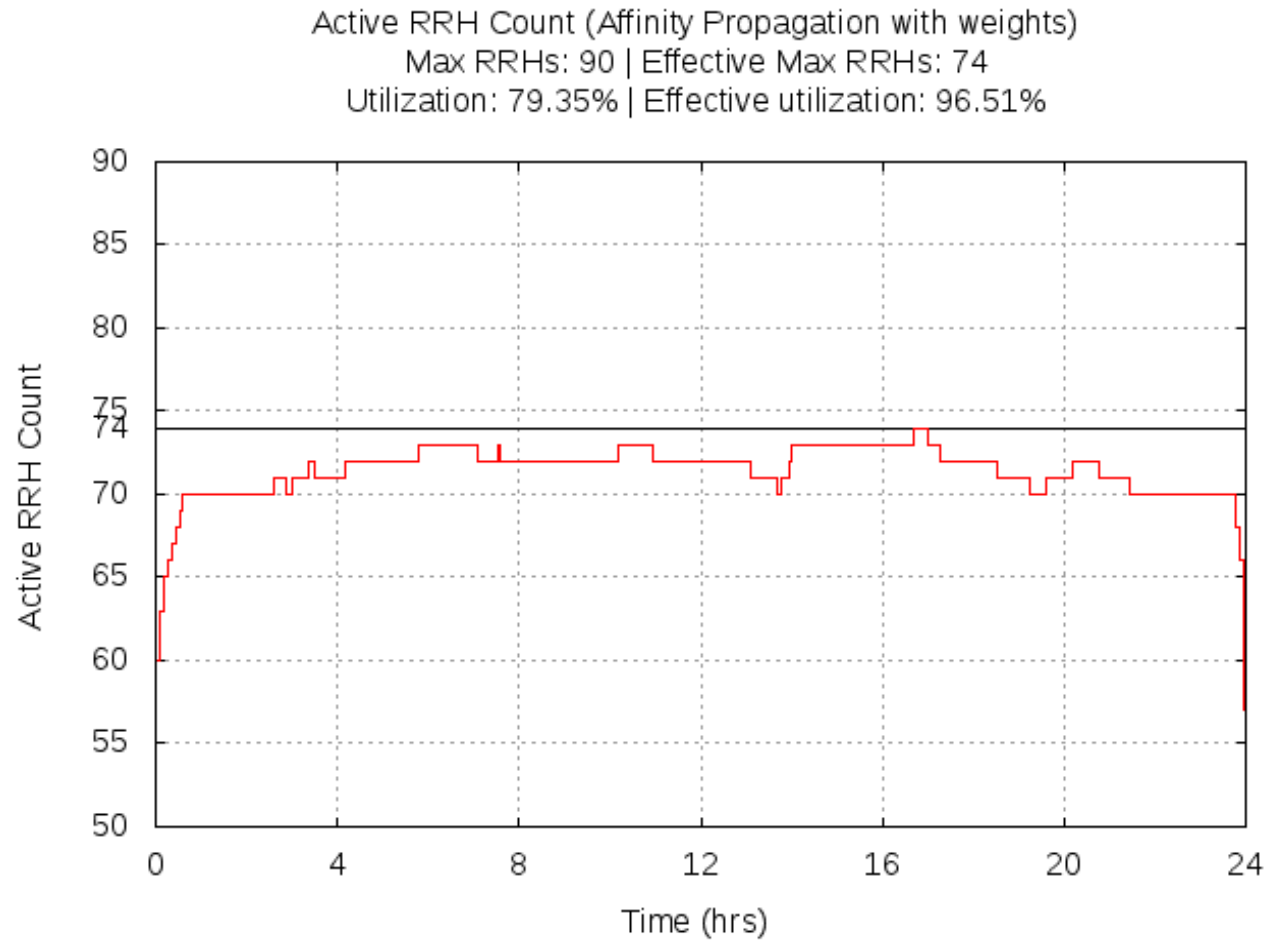

Fig. 10. Clusters fluctuation due to the affinity propagation with weights clustering. 
From these figures we can draw a parallel with the experiments where the researches manipulate the clusters of cellular towers to conserve the energy [14][13]. Our experiments also show the promise to reduce power consumption if towers' operating states can be manipulated without loss of coverage. For the given input we see about $80 \%$ of the RRH pool utilization which translates to roughly $20 \%$ of saving, and if we consider a scenario when the UE activity is extremely low, such as at night, this figure drops to the floor value corresponding to the number of clusters in the system. In this regard, the clustering method and consequently the size of the clusters can affect how much saving can be achieved during those quiescent hours. From TABLE III, assuming the worst case scenario when we can only have small clusters such as in the hierarchical clustering, the potential energy saving in the RRH pool is about $40 \%$. Again, we want to emphasize that in this paper we are assuming some towers within a cluster are capable to compensate for those that were switched off.

\section{B. Experiment I}

One of the potential advantages discussed by proponents for a centralization of radio networks is the overall improvement in performance for the tasks involving inter-tower cooperation such as handovers, interference mitigation techniques, CoMP, etc. [1][4][16][5]. In this part of our experiment we investigate how packing can affect the number of handovers that happen between clusters packed on different hosts. A UE handover involves migration of UE context between towers, which takes time and bandwidth, and in a centralized architecture, where the BBU pool aggregates multiple virtual towers and the internal network assumed to be shared between all of them, suboptimal BBU instance placement can result in unnecessary bandwidth increase due to handovers being passed between physically neighboring BBUs which happened to be served by different hosts.

In this experiment, we are using a hierarchical clustering algorithm to form the clusters (infigure abbr: HIER), and pack them using six different algorithms where only location-aware algorithms consider RRH physical position during packing calculation. We have three configurations for each packing experiment, where we vary the size of the host (4CPU and 5CPU) and the initial packing - location based (L.Init) and randomized (R.Init). For randomized

placement we just force 1-to-1 host-cluster mapping during initialization and let the packing algorithms deal with it. 
Figure 11 shows the percentage of how many handovers between clusters located on different hosts occur. We are not considering handovers within the cluster, since they are already on the same host, so, the percentage is relative to overall handovers between different clusters.

Inter-host handovers

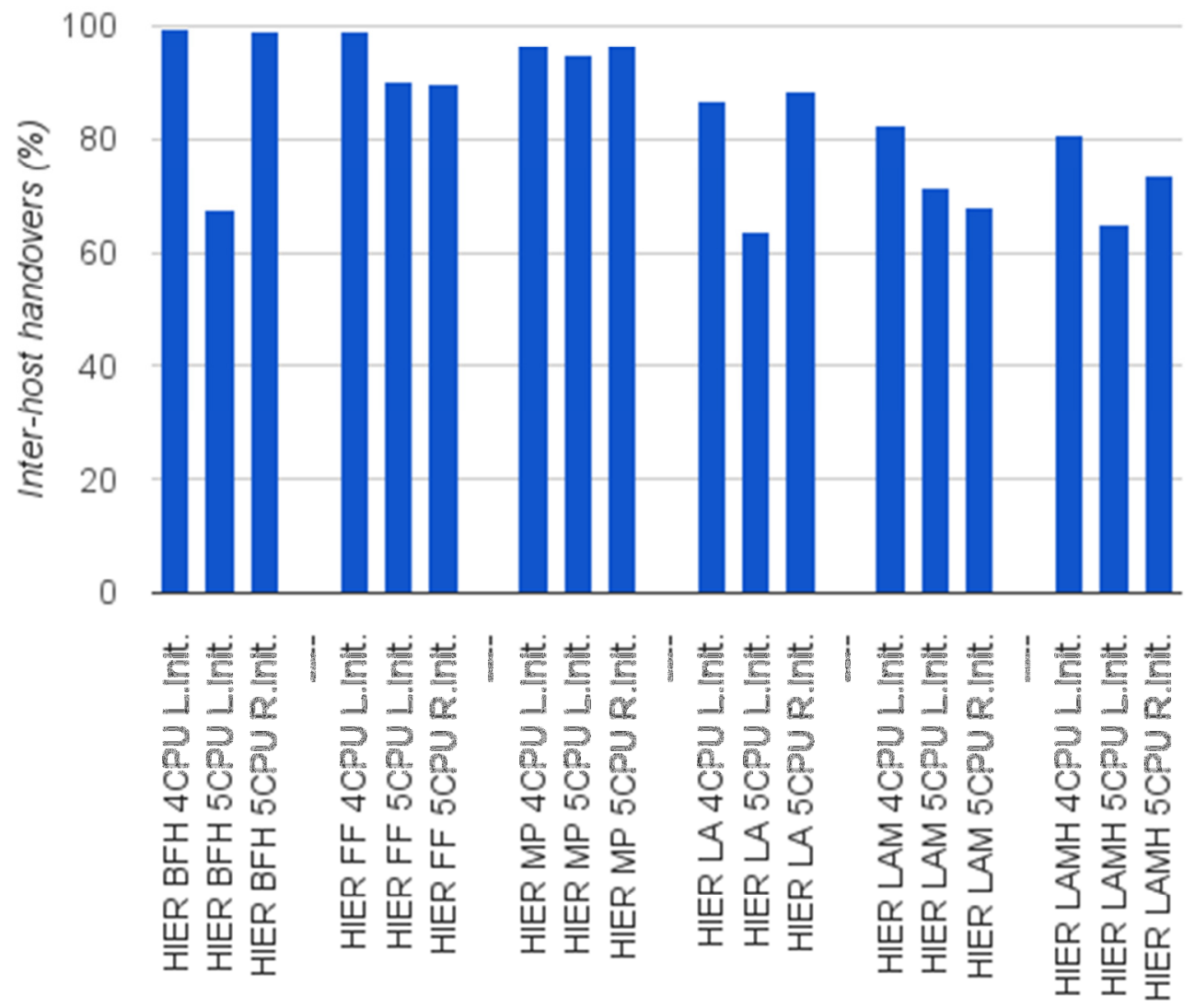

Fig. 11. Inter-host handovers.

We can see a general trend is that by adding a location-awareness to the packing algorithms the overall inter-host handovers improves. The graph shows several outliers of which "HIER BFH 5CPU L.Init." stands out the most and is just a good example of the fact that an algorithm without a location-awareness incorporated, also can produce a good result, although it is not quite as consistent across different simulations. The investigation of this result showed that there was a combination of three major factors that benefited the BFH in this simulation. First, we used a location based cluster placement during initialization. Secondly, the BFH by itself avoids any unnecessary migrations and is quite static if the resource utilization fluctuations are mild. 
Thirdly, Figure 9 confirms the fact that in this particular simulation, the input variation was probably not enough for the $\mathrm{BFH}$ algorithm to significantly affect the initial cluster packing.

One thing to note regarding location-aware algorithms is that they do not force the packing using whatever criteria they are based on. During the resource usage evaluation, the algorithms merely look up any "good" neighbors and if the resources are available only then the neighbors are brought together. This approach aims to put neighbors together, but at the same time, might cause a less tight packing.

\section{Experiment II}

For the second experiment we are running all six packing algorithms against clusters formed during initialization by three clustering algorithms. The purpose of this experiment is to investigate the performance of the algorithms discussed in this paper, identify potential problems and establish some facts from the results.

Since we are packing dynamic clusters of virtualized BBUs on hosts it has an effect on the host pool. Figure 12 depicts aggregated statistics for the pool collected during simulation, specifically - the global minimum of active hosts, the global average, and the global maximum. In this graph the most representative information is the average host count and its relation to the global maximum. Out of all experiments, for the current input, "HIER BFH" is the most well behaved with regard to the host utilization which is indicated by its average and maximum values being close. Nevertheless, as TABLE III indicates, all "HIER" type experiments deal with greater number of clusters, and we can see that it has been reflected with average host count being greater than in "AP*" types. 
Active Host Count Statistics

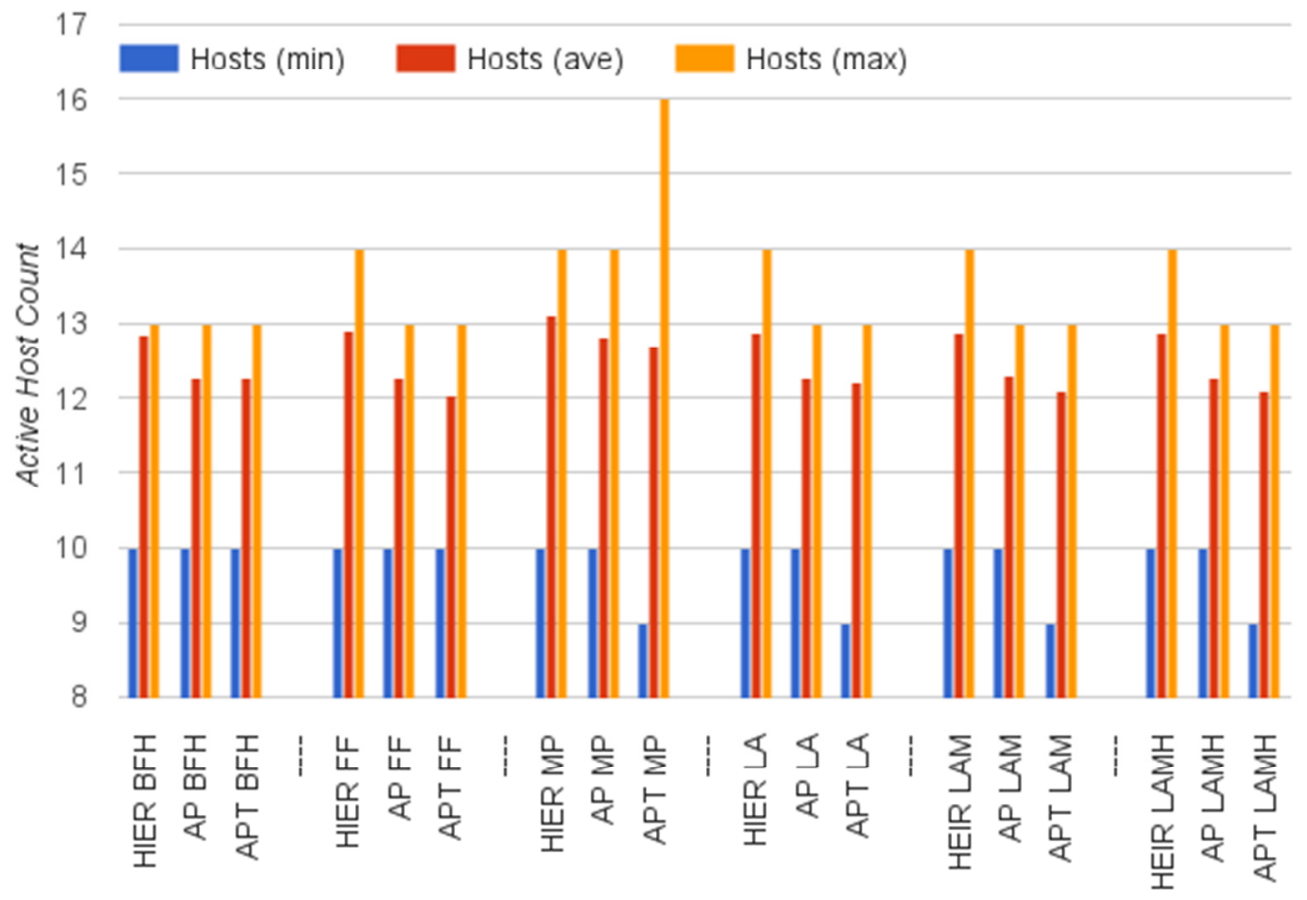

Fig. 12. Active host count runtime statistics.

All the experiments in this part take advantage of location-based initialization, and all the algorithms make extensive use of regression analysis of history data for resource usage. Because of that, the resource manager resizes and packs clusters tightly more often than not. The outcome of this is that the average runtime values for the host count in these experiments are the most representative for the resource utilization, and on Figure 13 we can see the bars corresponding to the estimated processing power follow the trend. 
Estimated Power (kW)

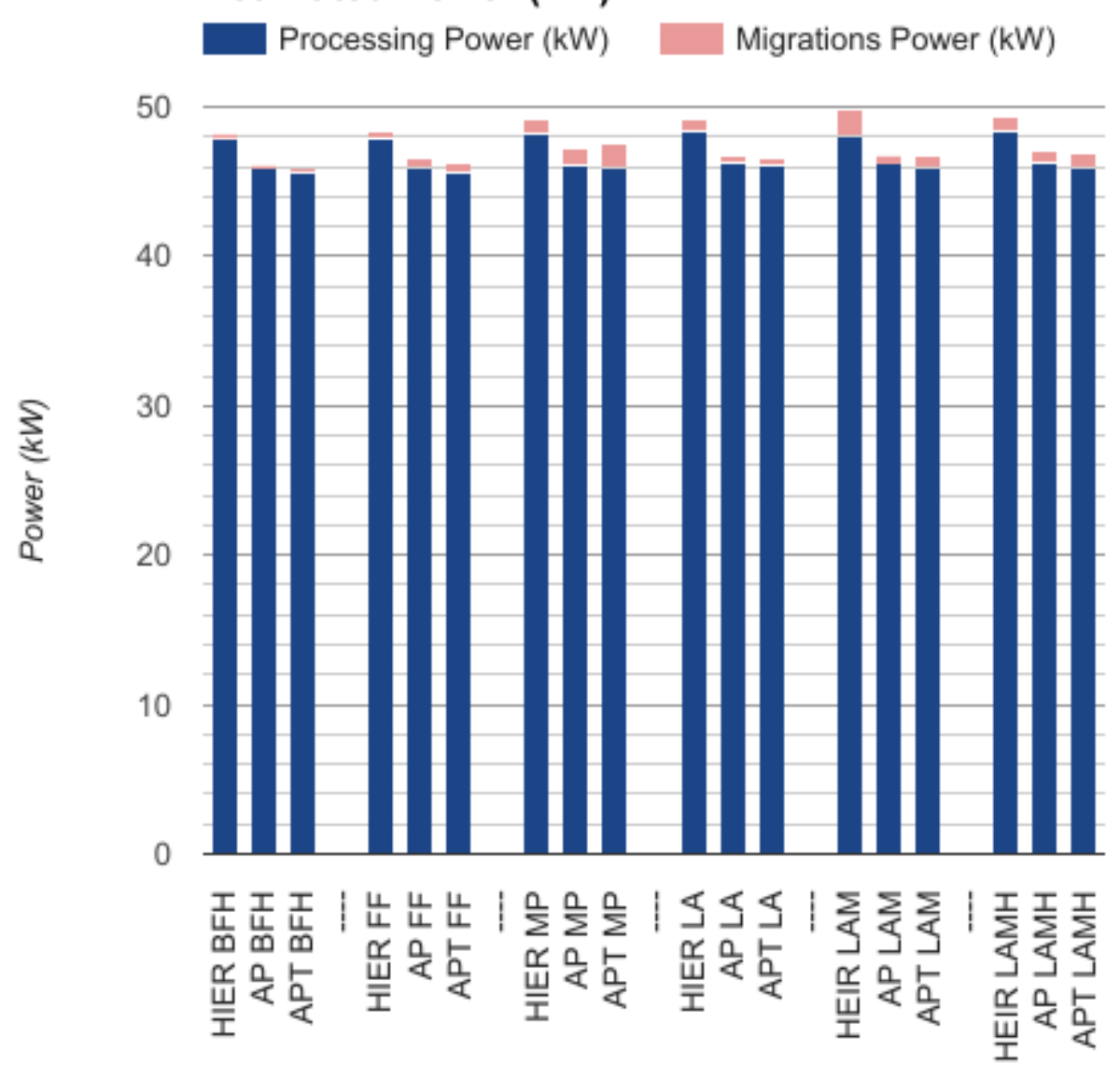

Fig. 13. Estimated power in the BBU pool.

Both - Figure 12 and Figure 13 - show the similar tendency to benefit clustering which results in a smaller set such as APT. The global minimum values in Figure 12 along with TABLE III allow us to extrapolate that during "off-peak" hours, the AP- and APT- based setups would have much better energy saving characteristics due to the single fact that they have fewer clusters. 
On the figure below we can see some experiments report an extensive use of migrations, so we further investigated this and found out that in some scenarios, the packing algorithms that have multiple criteria to make the decision can have some sort of a jitter which can lead to an excessive number of migrations as it can be observed on Figure 14 for "HIER LAM”, “AP MP”, and "APT MP" experiments.

Migrations

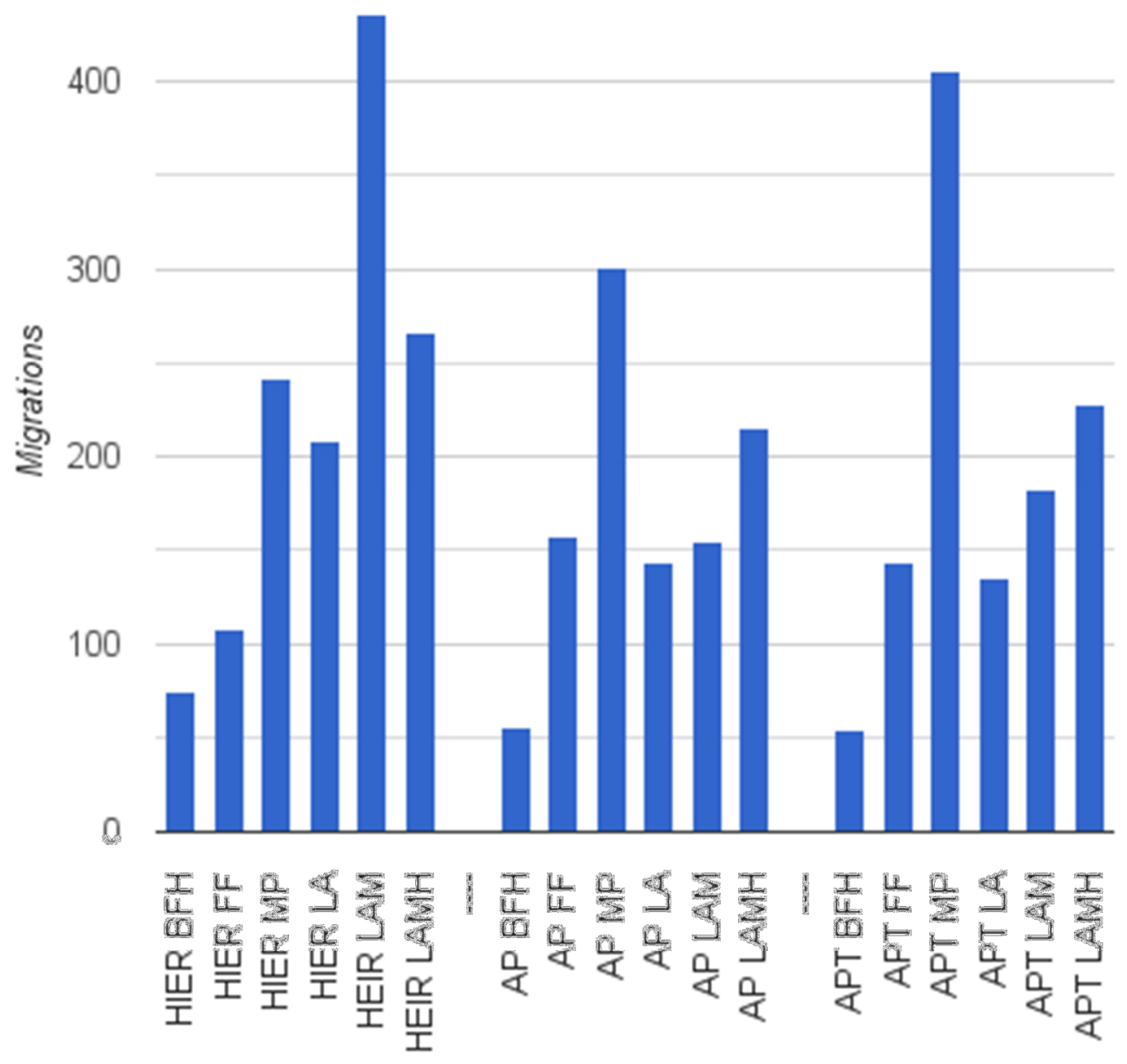

Fig. 14. Cluster migrations due to re-packing. 
All three experiments, upon deeper investigation, proved to be the extreme cases when the lack of priorities and restricting policies affects the decision making process resulting in suboptimal placement and jitter which can occur for example when there are multiple equipotent solutions to a packing problem. Compared to the $\mathrm{BFH}$ algorithm, our location-aware algorithms still have some room for improvement since a great number of migrations not only burdens the internal network, but also can affect the quality of service.

As in Experiment I, here we also gathered and examined the handover statistics. Figure 15 shows the handovers count during simulation for all packing and clustering algorithms.

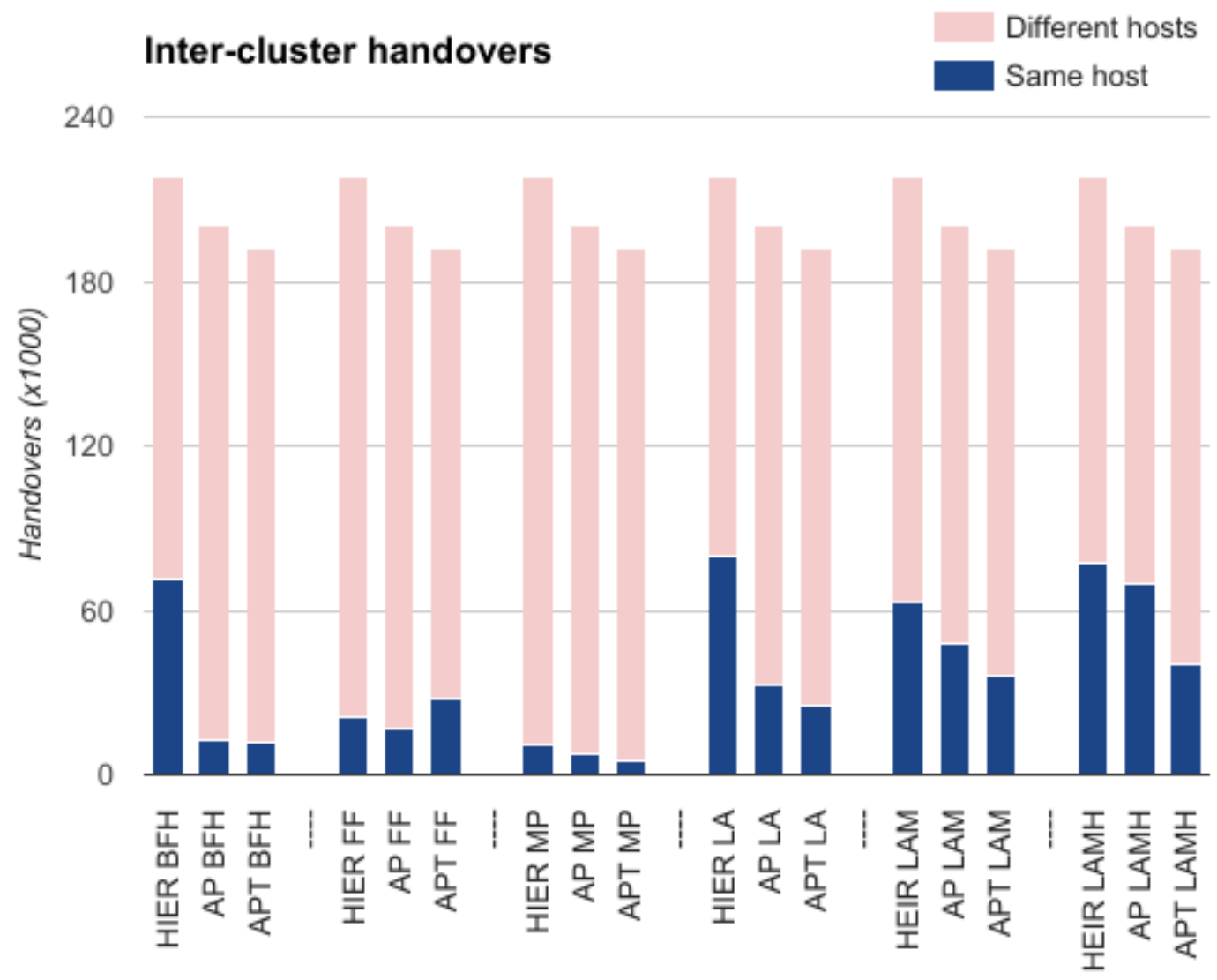

Fig. 15. Inter-cluster handovers.

The trend shown by the stacked bars in the figure reflect the fact that the number of intercluster handovers gets affected by the clustering algorithm. But also, we can see that having smaller clusters has it benefits since packing algorithms have better opportunity to arrange them in more handover-friendly manner. The evidence of this is more clearly shown in Figure 16 where we isolate inter-host handovers. In this figure we can trace and extrapolate two interesting 
features. 1) Having smaller clusters, even if their number increases as a result, positively affects inter-host handover count, especially when location-aware packing is used. 2) Enforcing specifics investigation during packing, beneficially impacts inter-host handovers as well - from the figure above we can see how using more location-specific properties to negotiate packing positively affects inter-host handover count.

\section{Inter-host handovers}

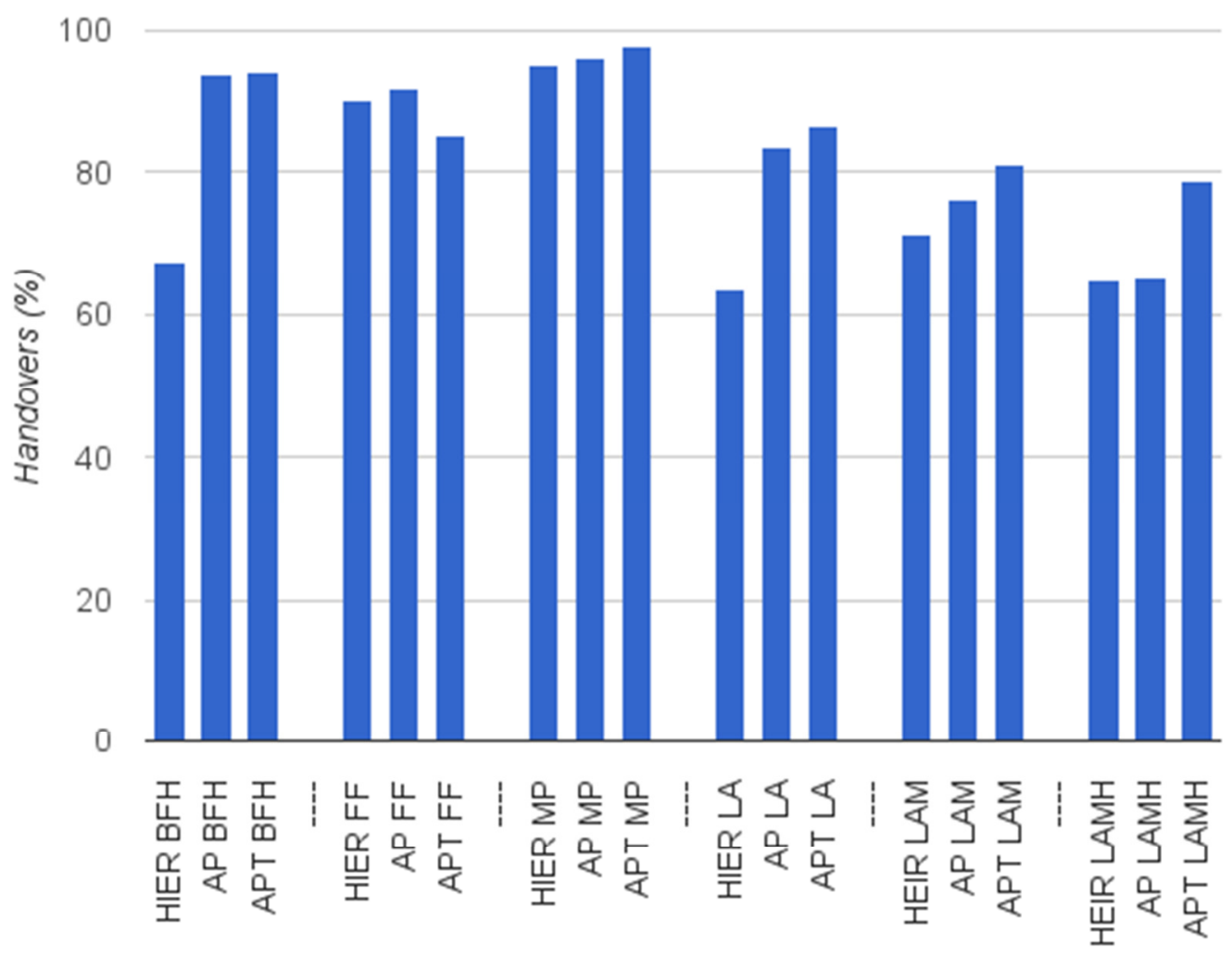

Fig. 16. Inter-host handovers.

There is one more thing we want to discuss, which is the host pool utilization. Figure 17 shows the effective host pool utilization, meaning, we have calculated here what the utilization advantage is compared to the runtime active host count maximum. The idea behind it is that we are comparing dynamic packing to the static packing when the resource manager estimates expected utilization peak, allocates the necessary resources and activates them for the full period. It has to be noted, that in this comparison we are looking at the most optimistic scenario for the static provisioning where the ceiling for utilization is based on a well-packed runtime maximum. 


\section{Overall host utilization}

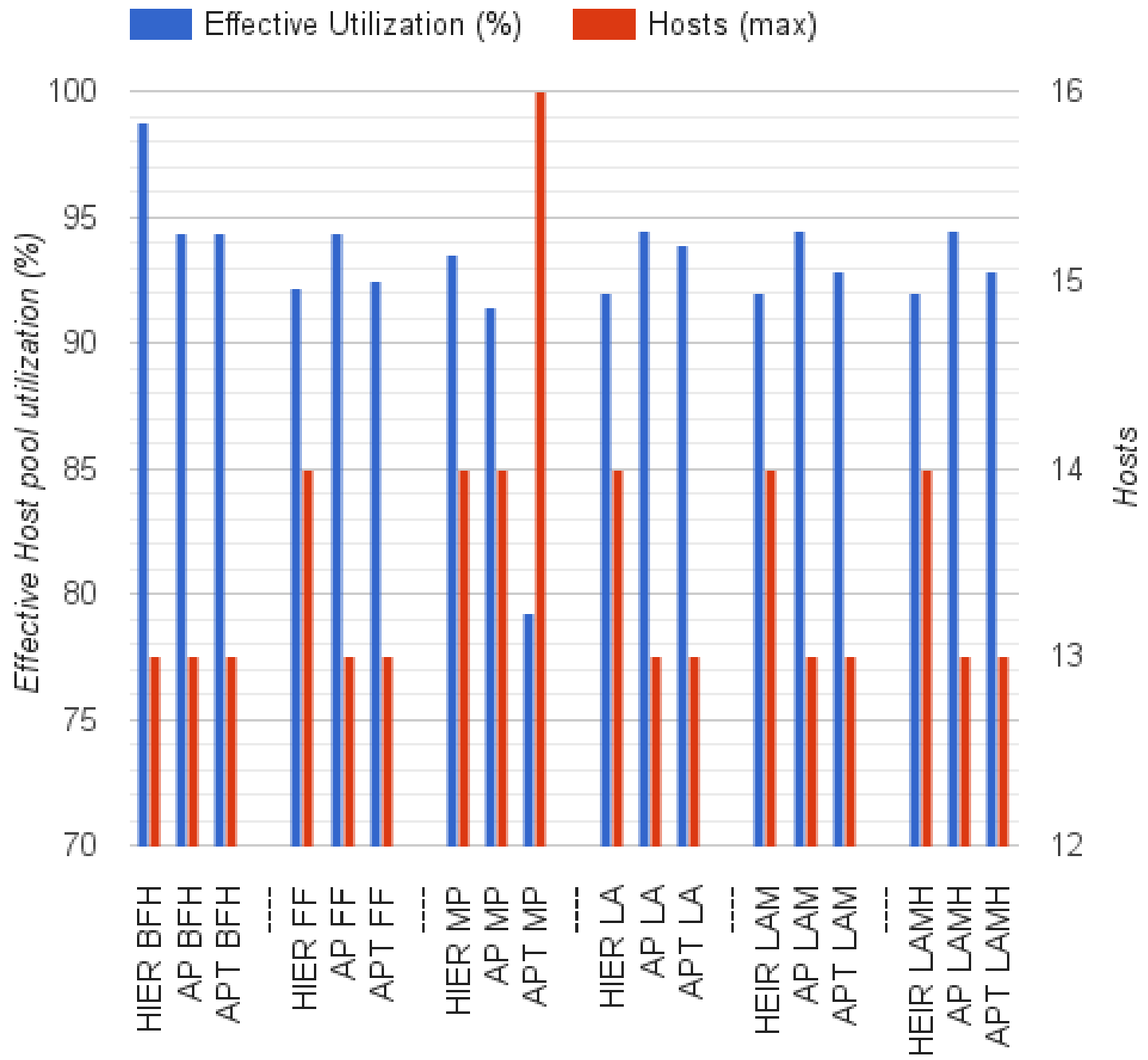

Fig. $17 . \quad$ Host pool utilization.

The purpose of the figure above is not to compare the algorithms against each other, rather we want to see the improvement in the host utilization if the alternative solution for the resource management is based on a static provisioning when the global maximum is assumed to be from a dynamic provisioning and the clusters are initialized on hosts and never overutilize their hosts during the runtime.

In the figure we see the improvement in the host pool utilization to be about $7 \%$ in average if we exclude two outliers - "HIER BFH" and "APT MP". In conjunction with previous results, these two special cases allow us to extrapolate the following. 1) If the clustering and packing algorithms effectively "cushion" the input fluctuation as it happened in "HIER BFH", the global 
maximum can be misleading for a static provisioner to make any assumptions about the peak. The evidence of that can be seen if we look at other "HIER" based experiments, where the global maximum is a bit higher. 2) "APT MP" experiment shows that at some point during the runtime the packing algorithm had a suboptimal packing configuration resulting in the host utilization spike. We can extrapolate that in a real life situation, this spike value would be more appropriate for a static provisioner to assume, especially if we look back at the clusters fluctuation waveform and note that for this experiment the clusters are mostly at $80 \%$ utilization on average and there is no guarantee that at some point the input wouldn't reach the ceiling. So we can expect the potential reasonable savings in the host pool utilization to be more notable than the average shown in the figure above.

TABLE IV. Clustering algorithms features.

\begin{tabular}{||l|l|l||}
\hline $\begin{array}{l}\text { Clustering } \\
\text { algorithms }\end{array}$ & Advantages & Disadvantages \\
\hline $\begin{array}{l}\text { Hierarchical } \\
\text { Clustering }\end{array}$ & $\begin{array}{l}\text { Inherently can cluster towers that share } \\
\text { common characteristics, e.g. intra-cluster } \\
\text { distance restriction. } \\
\text { Adding new criteria does not increase the } \\
\text { runtime complexity. }\end{array}$ & $\begin{array}{l}\text { Not flexible if the clustering } \\
\text { criteria includes } \\
\text { approximations or } \\
\text { assumptions. } \\
\text { Node traversal order affects } \\
\text { the resulting clusters. }\end{array}$ \\
\hline $\begin{array}{l}\text { Affinity } \\
\text { propagation }\end{array}$ & $\begin{array}{l}\text { Inherently forms clusters around existing } \\
\text { nodes. } \\
\text { Flexibility in adjusting clustering criteria: } \\
\text { selecting candidate centroids, size of the } \\
\text { cluster, applying custom cluster rules. }\end{array}$ & $\begin{array}{l}\text { Convergence time is not } \\
\text { deterministic and runtime } \\
\text { can fluctuate. }\end{array}$ \\
\hline $\begin{array}{l}\text { Affinity } \\
\text { propagation } \\
\text { [6] with } \\
\text { mobility }\end{array}$ & $\begin{array}{l}\text { Specialization by applying custom rules, in } \\
\text { this case, using historical data of UE } \\
\text { mobility. }\end{array}$ & $\begin{array}{l}\text { Increased convergence time } \\
\text { compared to normal Affinity } \\
\text { Propagation. }\end{array}$ \\
\hline
\end{tabular}


TABLE V. Packing algorithms features.

\begin{tabular}{||l|l|l|}
\hline $\begin{array}{l}\text { Packing } \\
\text { algorithms }\end{array}$ & Advantages & Disadvantages \\
\hline BFH [7] & Tight packing. & $\begin{array}{l}\text { Without specialization can result } \\
\text { in increased inter-host } \\
\text { bandwidth utilization due to UE } \\
\text { migrations. }\end{array}$ \\
\hline FF [8] & $\begin{array}{l}\text { Fastest out of all used algorithms used } \\
\text { in this experiment. }\end{array}$ & $\begin{array}{l}\text { Same as in BFH. } \\
\text { Sub-optimal packing. }\end{array}$ \\
\hline MP [5] & $\begin{array}{l}\text { Allows packing clusters that } \\
\text { complement each other which, when } \\
\text { grouped, exhibit the overall resource } \\
\text { requirements to be near flat-line in the } \\
\text { course of the selected period. }\end{array}$ & $\begin{array}{l}\text { Requires history analysis and } \\
\text { pattern recognition. } \\
\text { Can result in increased inter- } \\
\text { host bandwidth utilization due } \\
\text { to UE migration if neglected. }\end{array}$ \\
& & $\begin{array}{l}\text { False positives can cause sub- } \\
\text { optimal packing. }\end{array}$ \\
\hline $\begin{array}{l}\text { LA (and its } \\
\text { variants - } \\
\text { LAM, LAMH) }\end{array}$ & $\begin{array}{l}\text { Specialization. } \\
\text { Targets the mobility characteristic } \\
\text { which affects bandwidth utilization } \\
\text { within BBU Pool. }\end{array}$ & $\begin{array}{l}\text { Requires history analysis and } \\
\text { pattern recognition. } \\
\text { Sub-optimal packing can be } \\
\text { introduced to meet the } \\
\text { specialization and target } \\
\text { requirements. }\end{array}$ \\
\hline
\end{tabular}

\section{CONCLUSION}

Centralized radio access network architecture is an actively researched topic and there are multiple implementation aspects that has to be addressed before it becomes more widespread. In this paper we are making several assumptions about capabilities of this architecture which are in accordance with numerous publications on the subject:

- a fronthaul can span a few dozen of kilometers,

- general purpose platforms are capable to host multiple BBU instances,

- migration of BBU instances between the hosts is feasible without the degradation of service,

- RRH's operating state can be remotely manipulated,

- RRH's zones of responsibility can be dynamically adjusted affecting its power and coverage area. 
In our experiments the main focus was on BBU instances, which we organized into clusters and placed them on hosts using three clustering and six packing algorithms. Our goals were to adapt the packing algorithms from data centers, investigate their behavior in the mobile environment and, based on the results, propose alternative solutions and recommendations.

Dynamically manipulating clusters of BBU instances based on demand from UEs has a positive effect on RRH pool as well, given that there is a one-to-one RRH-BBU mapping and their operation states are synchronized, i.e. when a BBU is turned "off", the corresponding RRH transitions to a dormant state as well. Our results also showed savings in power usage within the RRH pool, even for scenarios when variations in the UE usage pattern are not extensive during active hours [13][14].

Adapting packing algorithms from data centers to manage BBU instances within a BBU pool improved the overall energy efficiency and resource utilization [7][8]. For example the BFH algorithm showed the best result packing the clusters on fewer hosts with the lowest number of migrations. But, the lack of a mechanism to account for mobility information introduced the overhead caused by the increased inter-host traffic associated with UE handovers. Furthermore, during the "peak" hours, the performance of the mobile network (e.g. QoS) might be prioritized over energy conservation, thus a packing that reduces inter-host traffic associated with interBBU cooperation would be more beneficial. In this paper we introduced a location-aware algorithm and two modifications that target more specific aspects of mobile environment which potentially can help to address the issue of increased inter-host traffic.

Our experiments showed that packing algorithms adapted from data centers can be improved with the regard to inter-host traffic by simply applying location-aware initialization during initial packing, although, it does not guarantee the consistency across the runs. In this regard, locationaware algorithms showed more promise to consistently reduce inter-host traffic by sacrificing the packing efficiency. From the experiments we can outline a few guidelines to address packing problem in the mobile environment.

- Avoid generic solutions being applied across various regions - each area requires finetuning to address its peculiarities. 
- Avoid random initializations, rather make use of historical and locality information to perform the most optimal initial placement.

- For different periods of the day, identify invariant base sets of clusters that can be and should be locked to their hosts in order to avoid the increase of inter-host traffic if they happened to be migrated to another host caused by suboptimal false-positive placement.

- Packing of clusters in a mobile environment requires the inspection of multiple runtime and historical parameters some of which might contradict each other. As a result, it is essential to incorporate enforcing policies that would allow for more intelligent decision making when the algorithm has to pick among equally potent solutions.

- "Mix and match". Having a centralized location with a vast processing power allows a resource manager to run several packing algorithms in parallel and pick the one that fits the runtime criteria.

In this paper we picked handovers as a measuring stick for our algorithms to address a potentially critical problem when BBU instances are brought together under the same roof and share the networking infrastructure - if the dynamic packing algorithm does not account for the locality at least, they can be accidentally packed in the worst manner when all of the inter-BBU traffic goes thru the network. Resource management in a BBU pool has its own challenges which are not present in data centers, thus, it is essential to identify the key properties which should be addressed by any algorithm introduced into the system in order to avoid the degradation of service. 


\section{REFERENCES}

[1] A.Checko, H.L. Christiansen, Y.Yan, L.Scolari,G.Kardaras, M.S.Berger,L.Dittmann, "Cloud RAN for Mobile Networks-A Technology Overview", IEEE Communication Survey\&Tutorials, Vol.17, No.1, 1st Quarter 2015, pp.405-426.

[2] A. Virdis, G. Stea, G. Nardini, "SimuLTE - A Modular System-level Simulator for LTE/LTE-A Networks based on OMNeT++," Proc. 4th International Conference on Simulation and Modeling Methodologies Technologies and Applications (SIMULTECH 2014), Aug. 2014.

[3] C. De Sa, "Basic Collision Detection in 2D", http://devmag.org.za/2009/04/13/basiccollision-detection-in-2d-part-1/, Apr. 2009.

[4] D. Lee et al., "Coordinated multipoint transmission and reception in LTE-advanced: deployment scenarios and operational challenges," in IEEE Communications Magazine, vol. 50, no. 2, pp. 148-155, February 2012.

[5] D. Pompili, A. Hajisami and T. X. Tran, "Elastic resource utilization framework for high capacity and energy efficiency in cloud RAN," in IEEE Communications Magazine, vol. 54, no. 1, pp. 26-32, January 2016.

[6] Frey BJ, Dueck D, "Clustering by passing messages between data points," Science 315(5814):972-976, 2007.

[7] J. Huang, K. Wu and M. Moh, "Dynamic Virtual Machine migration algorithms using enhanced energy consumption model for green cloud data centers," 2014 International Conference on High Performance Computing \& Simulation (HPCS), Bologna, 2014, pp. 902910.

[8] J. Liu, J. Guo and D. Ma, "Traffic Aware Virtual Machine Packing in Cloud Data Centers," 2016 IEEE 2nd International Conference on Big Data Security on Cloud (BigDataSecurity), IEEE International Conference on High Performance and Smart Computing (HPSC), and IEEE International Conference on Intelligent Data and Security (IDS), New York, NY, 2016, pp. 256-261.

[9] K. Alexandris, N. Nikaein, R. Knopp and C. Bonnet, "Analyzing X2 handover in LTE/LTEA," 2016 14th International Symposium on Modeling and Optimization in Mobile, Ad Hoc, and Wireless Networks (WiOpt), Tempe, AZ, 2016, pp. 1-7.

[10] Navid Nikaein, "Processing Radio Access Network Functions in the Cloud: Critical Issues and Modeling," Proceedings of the 6th International Workshop on Mobile Cloud Computing and Services, September 11-11, 2015, Paris, France.

[11] R. N. Calheiros, R. Ranjan, A. Beloglazov, C. A. F. De Rose, and R. Buyya, "CloudSim: a toolkit for modeling and simulation of cloud computing environments and evaluation of resource provisioning algorithms," Software-Practice and Experience, v. 41, pp. 23-50, 2011.

[12] S. Pelley, D. Meisner, T.F. Wenisch, J. VanGilder, "Understanding and abstracting total datacenter power," in: Workshop on Energy-Efficient Design, 2009.

[13] S.Zou, Y.Zhang, Y.Tang, "Resource Allocation Mechanism Based on Two-Step Mapping for Saving Energy in Wireless Network Virtualization", IEEE 9th International Conference on Anti-counterfeiting, Security, and Identification, 2015, pp. 150-154.

[14] Sang Hyun Lee, Illsoo Sohn, "Affinity Propagation for Energy-Efficient BS Operations in Green Cellular Networks", Wireless Communications IEEE Transactions on, vol. 14, pp. 4534-4545, 2015, ISSN 1536-1276. 
[15] Sourjya Bhaumik, Shoban Preeth Chandrabose, Manjunath Kashyap Jataprolu, Gautam Kumar, Anand Muralidhar, Paul Polakos, Vikram Srinivasan, Thomas Woo, "CloudIQ: a framework for processing base stations in a data center", Proceedings of the 18th annual international conference on Mobile computing and networking, August 22-26, 2012, Istanbul, Turkey.

[16] "C-RAN The Road Towards Green RAN", China Mobile Research Institute, Beijing, China, Oct. 2011, Tech. Rep.

[17] J. Huang, K. Wu, and M. Moh, "Dynamic virtual machine migration algorithms using enhanced energy consumption model for green cloud data centers," 2014 International Conference on High Performance Computing and Simulation (HPCS). 


\section{APPENDIX A: SIMULATOR IMPLEMENTATION}

To run the experiments we built a high-level behavioral event-driven application to simulate BBU pool, RRHs and mobile UEs. We looked at various simulation platforms before committing to develop our own and had to discard them with a single reason being their accuracy. It is inevitable for any simulation tool with the increased accuracy and adherence to the real-life implementation to have the runtime speed significantly reduced and consequently the turnaround time for the results increased. LTE targeted simulators such as SimuLTE or MatLab-based Vienna LTE-A Simulators are tailored towards accurate simulation of LTE architecture's underlying protocols from application layer down to physical layer of the stack which in turn has an adverse effect on a simulation time. We also looked at CloudSim platform, a modeling tool for data centers, and while we discarded it as our base platform, we borrowed some of the ideas used in its implementation [11].

The diagram on Figure 18 shows the essential components in our implementation along with their logical association with a BBU pool and open world.

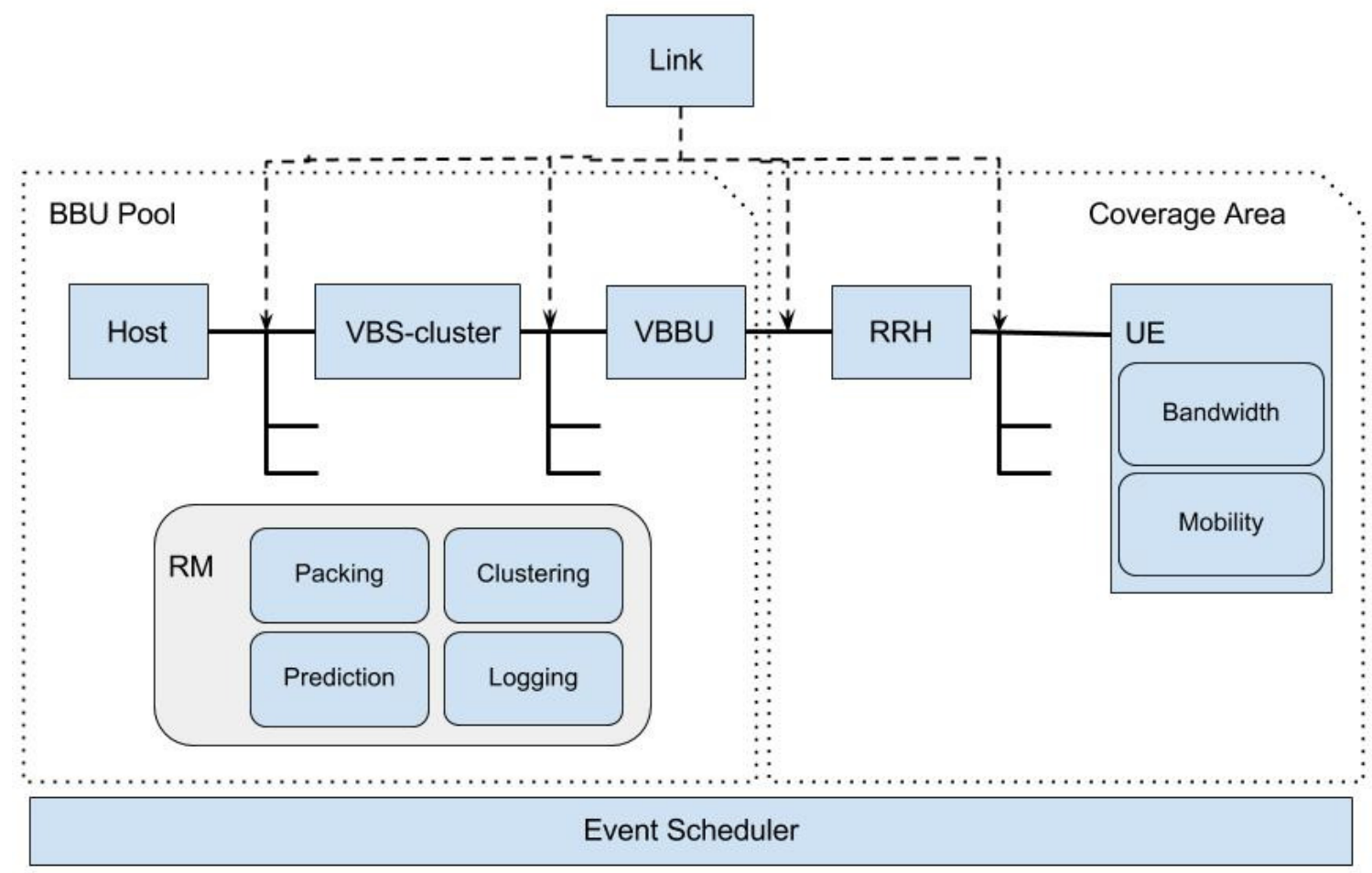

Fig. 18. CRANSim block diagram 
In the BBU pool we maintain the array of Hosts which can be activated and deactivated on demand. Each Host can serve multiple VBS-cluster instances depending on available resources (specified by the configuration) and a VBS-cluster's size which can be represented by CPU, memory, storage and bandwidth utilization. When we initialize a simulation, a clustering algorithm is applied on VBBU-RRH pairs (VBSs) which groups them and places them into VBS-clusters. Instances of RRHs and UEs are part of the outside world - coverage area, with RRHs being stationary at the designated coordinates, and UEs being the main source of the events during the runtime due to their randomized nature expressed through mobility and bandwidth requests. Main resource management (RM) tasks within the BBU pool are packing, clustering, prediction and logging, etc. Logging functionality encompasses monitoring and storing essential runtime system information which can be used for debugging, future runs as the input into the prediction component or to generate reports.

"Link" block in Figure 18 represents an abstraction for communication channels between the components. Within the links we can enforce bandwidth allocation policy which is necessary to negotiate individual channel bandwidth. By default, the bandwidth is equally distributed among the downlinks unless some require less with the remainder being redistributed among those downlinks requesting more. The topology of components create a tree of chained links rooting at the BBU pool level, and when the bandwidth request is created at a particular node, it creates a 'rippling' effect of requests up to the root and back down to all the nodes which get affected. We have implemented several optimizations that reduce the impact of the 'ripple' by detecting and skipping redundant recalculations.

The event scheduler takes the form of a queue with the time granularity set to 1 millisecond. The events coming from various components are placed into individual queues (frames) associated with particular time slots. This setup improves the insertion, lookup and removal of events from the global queue by keeping it to a manageable size. Further, it simplifies processing of events that occur concurrently ( sharing the same time slot ) which also facilitated by the inherent hierarchical topology of the main simulated components (UEs, RRHs, VBBUs, etc.). Figure 19 shows an example how a frame for a selected time slot is processed. When the global queue selects a frame at the front, the frame is dequeued and the scheduler invokes the events in whatever order they were inserted into the frame (label 1). When the currently processed event 
generates a new event that must be scheduled for an immediate execution, the scheduler inserts it right at the front of the global queue with the same timestamp (label 2). And if some event wants to register a new event with a delay, the scheduler performs a binary insertion at the requested time position (label 3).

\section{Global queue}

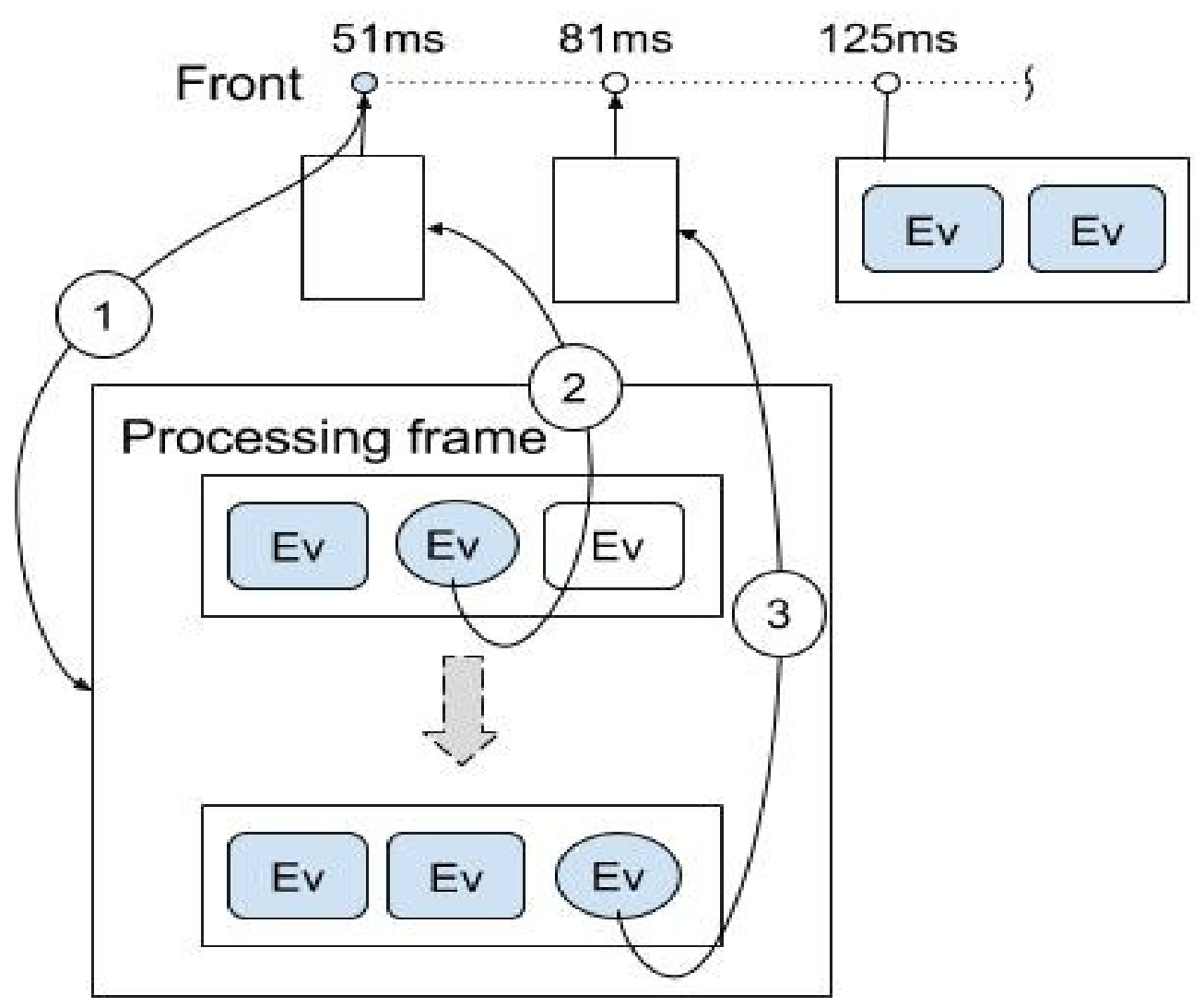

Fig. 19. Scheduler's global queue processing.

The main source of events for the simulation is UE. Each UE can request a certain bandwidth for a particular duration of time by creating a bandwidth request event for the uplink channel connected to the RRH in range. Also, some UEs move actively across the coverage area generating numerous movement events at which point the mobility functionality not only calculates the displacement, but also triggers connectivity and handover events. Handover also can be the source of bandwidth recalculation events - when a UE detaches from a RRH, it releases any bandwidth used, and then has to request for a bandwidth allocation when it attaches to another RRH. 
The movement processing is one of the most expensive functions in this simulator. When UE moves, it has to maintain in- and out-of-range state with a neighboring RRH. In our implementation we tried two approaches to perform mobility tracking - a time sampling and a trajectory pre-calculation. For the sample-based method we used 500 millisecond intervals when the UE has to verify its proximity and either attach or detach to the closest RRH. This method proved to be extremely costly for the simulations spanning a 24 hour period with the UE pool measured in thousands. To address this performance issue we attempted to use a trajectory precalculation technique based on collision detection techniques used in a video game development [3]. During initialization we parse UE's trajectory and perform path segmentation at the key points when UE crosses the regions covered by RRHs, then, for each segment we create a list of the in-range RRHs which further improves the performance since the number of RRHs to check for proximity is greatly reduced. The downside of this approach is that it requires a lot more memory to run the simulation. 